\title{
Memories of Sir Michael Atiyah
}

\section{Nigel Hitchin, Editor}

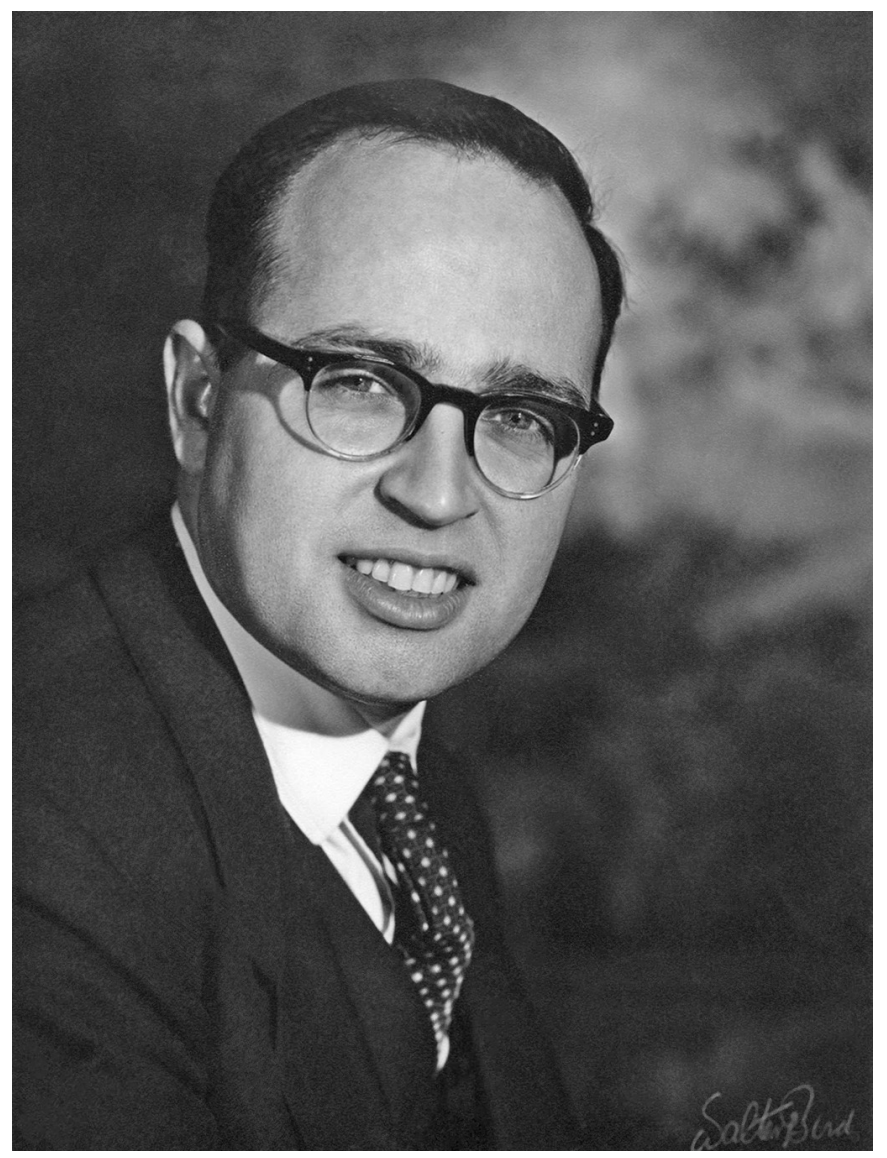

Figure 1.

Sir Michael Atiyah, a hugely influential figure in mathematics, died on January 11, 2019. A tribute appeared in the November 2019 issue of the Notices, but what follows is a col-

This is Part 2 of a two-part series on Sir Michael Atiyah. Part 1 was included in the November issue.

Communicated by Notices Associate Editor Chikako Mese.

For permission to reprint this article, please contact:

reprint-permission@ams . org.

DOI: https://doi.org/10.1090/noti1997 lection of more personal recollections-what it was like to be his student, to work alongside him, to have avenues of exploration pointed out, or to be inspired and energized by his unique personality. The contributions cover a full fifty years in which his interests moved from mathematics to incursions into physics. They are arranged in chronological order.

\section{Michael Atiyah and Representation Theory}

\section{George Lusztig}

I was very fortunate to have the opportunity to study with Michael for two months in Oxford in 1968 and for two years (1969-71) at the Institute for Advanced Study in Princeton, where he arranged for me to be invited. Michael Atiyah is famous for his work in algebraic geometry, algebraic topology, index theory, and, later, physics, but on the side he had important contributions to representation theory. I will try to explain some of them here.

Michael's 1967 paper with Bott contains a proof of the holomorphic Lefschetz fixed point formula that provides a wonderfully simple explanation for Weyl's character formula for $\operatorname{tr}(g, V)$ ( $g$ is a regular semisimple element, and $V$ is an irreducible rational representation of a complex semisimple group $G$ ). The explanation was in terms of the (finite) fixed point set of the automorphism defined by $g$ on the flag manifold of $G$. This was a model for me when I later worked with Deligne on representation theory of finite reductive groups, where the holomorphic Lefschetz fixed point formula was replaced by a Lefschetz fixed point formula in $l$-adic cohomology for certain automorphisms of finite order of a flag manifold and certain subvarieties

George Lusztig is the Abdun Nor Professor of Mathematics at MIT. His email address is gyuri@mit.edu. 
of it.

Michael's 1968 paper "The index of elliptic operators, III" with Singer contains an explicit formula (see 4.6) for $\operatorname{tr}(g, V)$ in which $g \in G$ is of finite order but not necessarily regular. While $\operatorname{tr}(g, V)$ could in principle be determined from the analogous traces with $g$ regular semisimple, this paper gives for the first time an explicit formula for $\operatorname{tr}(g, V)$ as a sum of contributions of the connected components of the fixed point set of $g$ on the flag manifold of $G$. This is a very interesting complement to Weyl's character formula.

Michael's 1977 paper with Schmid provides a new perspective on the discrete series of real semisimple groups based on the $L^{2}$-index theorem (a version of the index theorem for noncompact manifolds).

When I was Michael's student I was mainly working on problems in algebraic topology and index theory, but I also learned many things in representation theory from Michael, and eventually representation theory became my main focus.

Around 1971, I attended a talk by Quillen at the IAS, where he explained his solution of a conjecture of F. Adams in algebraic topology, which surprisingly used the modular and complex representations of the finite group $\mathrm{GL}_{n}\left(\mathbf{F}_{q}\right)$. After the talk I asked Michael whether the complex irreducible representations of $\mathrm{GL}_{n}\left(\mathbf{F}_{q}\right)$ were known. He told me that the characters were determined by J. A. Green, but the representations themselves were not understood. This seemed to be an interesting question, and thus I became more and more involved in representation theory (probably to the disappointment of Michael). My first job after Princeton, which I got, I believe, with some help from Michael, was at the University of Warwick, where J. A. Green was teaching.

After going to Warwick, I often visited Michael in Oxford, where he returned in 1972. On one of those visits he told me about a beautiful formula in which the $k$ th Adams operation $\psi^{k}(V)$ applied to the standard representation $V$ of $\mathrm{GL}_{n}(\mathbf{C})$ is expressed as an explicit alternating sum of irreducible representations of $\mathrm{GL}_{n}(\mathbf{C})$. Understanding this formula became one of the motivations for my work (in 1973) with Roger Carter on the modular representations of $\mathrm{GL}_{n}$. In our 1974 joint paper we give, among other things, a refinement of Michael's formula in which $\mathbf{C}$ is replaced by $\overline{\mathbf{F}}_{p}$, an algebraic closure of the field with $p$ elements, and $k=p$. In this case $\psi^{k}(V)$ becomes the Frobenius twist of $V$, and our refinement gave a resolution of $\psi^{k}(V)$ by Weyl modules (predicted by Michael's formula) that are linked with each other by an action of the affine Weyl group. This paper gave me later some support for stating a conjectural formula for the irreducible modular representations of a reductive group over $\overline{\mathbf{F}}_{p}$ in terms of

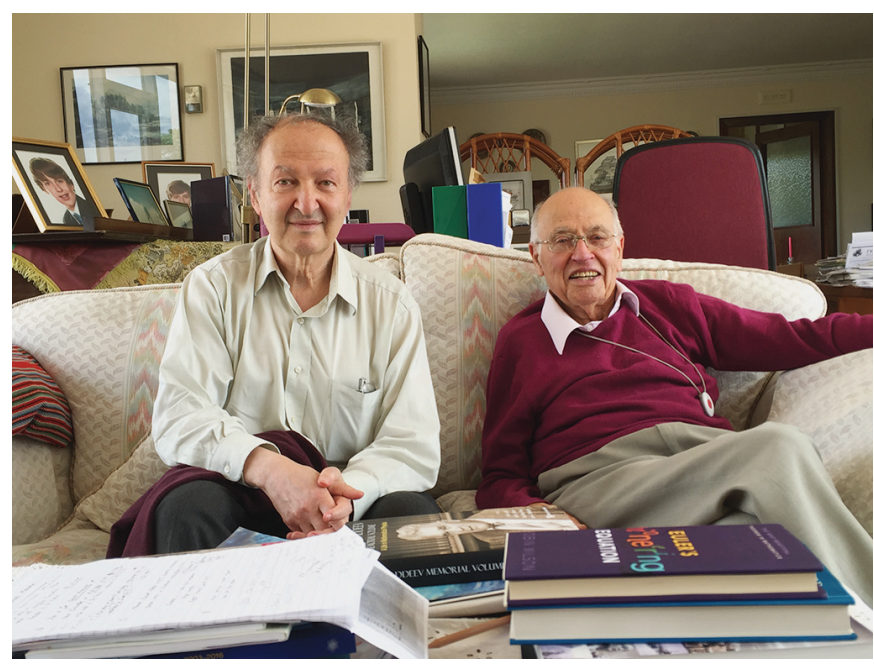

Figure 2. Lusztig and Atiyah in Edinburgh, June 2018.

the affine Weyl group.

One of the things that I learned as Michael's student was to use topological $K$-theory and its equivariant version, a theory very closely associated with Michael's work. In the early 1980s I used this in connection with an affine Hecke algebra $H$ by realizing the principal series representations of $H$ in terms of suitable equivariant $K$-theory of a flag manifold in which the parameter $q$ of the Hecke algebra becomes the standard generator of the representation ring of the circle group. This led me to a conjecture in which the representations of $H$ are realized in terms of equivariant $K$-theory of certain Springer fibers. (This conjecture was later proved by Kazhdan and me, thus establishing the part of the local Langlands conjecture which involves $H$. Here Michael's equivariant $K$-homology played a key role.)

I last saw Michael in June 2018 when, with my wife, I visited him at his apartment in Edinburgh. We had a wonderful time. I told him that we were staying in a hotel next to the statue of Maxwell (the existence of this statue is largely due to Michael's efforts). Michael told us about his admiration for Maxwell, whom he put on the same level as Newton and Einstein. He also explained some of his new mathematical ideas.

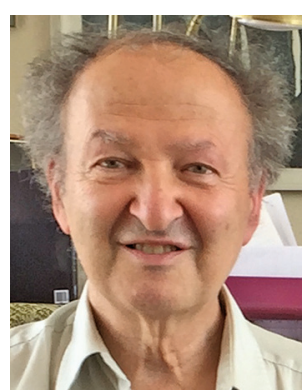

George Lusztig 


\section{Instantons and Monopoles}

\section{Nigel Hitchin}

Atiyah's most celebrated contribution to mathematics is the index theorem. Indeed the papers occupy two whole volumes of his collected works covering the twenty years since the Bulletin of the AMS published the initial paper in 1963. Louis Nirenberg recalls one of the seminars Atiyah gave at NYU at the time, which he began with the words: "Whenever I come here I seem to talk about the index theorem...," whereupon Nirenberg replied, "... and you are going to do it till you get it right!" (echoing the old story about the young soprano wondering why she was asked for so many encores). Yet, however powerful the index theorem was, it would be wrong to think of him carrying around this huge weapon and firing it at every opportunity. In fact, in what was probably his final mathematical paper [1], he deliberately avoided the more obvious application of the Atiyah-Patodi-Singer version for a more direct approach.

His choice of mathematical problems was more a recognition that they resonated with something he was familiar with. This was apparent in the work on instantons and monopoles in the 1970s and ' 80 s when I became less of a witness to his mathematical output and more of a participant. Singer brought the problem of solving the selfdual Yang-Mills equations to Oxford in 1977 from his contact with physicists at MIT. The question was one of finding, up to gauge equivalence, all connections on a principal SU(2)-bundle over $\mathbf{R}^{4}$ that minimize the $L^{2}$ norm of the curvature. With appropriate decay at infinity (which was confirmed later by Uhlenbeck's work) this became by conformal invariance a problem on the 4-sphere, a compact manifold where both manifold and bundle have nontrivial characteristic classes.

The index theorem did then provide a starting point, giving the local structure of the moduli space [2] and showing that there were more solutions than the ones provided to us by the physicists. But Michael's real interest was in using the ideas of Roger Penrose and his student Richard Ward to find the solutions in concrete form. Here characteristically he saw an opening into the type of geometry that he had enjoyed since the beginning: both he and Penrose had been exposed as undergraduates in Cambridge to the classical geometry of describing the lines in complex projective 3-space by the points in a four-dimensional complex quadric, the Klein quadric. This was the corner-

Nigel Hitchin is an Emeritus Savilian Professor of Geometry at the University of Oxford. His email address is hitchin@maths.ox.ac.uk. stone of Penrose's twistor theory, trading differential geometry in four dimensions for algebraic geometry in three. But everything was over the complex numbers, whereas the Yang-Mills problem concerned the 4-sphere.

So Atiyah first showed how twistor theory could be adapted to this situation by regarding complex projective 3-space as a real algebraic variety with no real points but with real lines. With this viewpoint the projective space fibered over the 4-sphere (and was even part of a more general picture for even-dimensional spheres). He then used the modern work in algebraic geometry of Horrocks, Barth, and others to give an explicit construction of the holomorphic vector bundles defining instantons. This followed from studying the structure of a module over a ring of polynomials generated by some sheaf cohomology groups associated to the vector bundle. If a certain sheaf cohomology group vanished, then the construction followed from essentially a single matrix of linear forms. But this was where the twistor theory came into play again, as it translated back to a simple question about a differential operator on the sphere. The fertile common ground between algebraic geometry and differential geometry that was in evidence here was dear to his own interests, not just classical algebraic geometry but also going back to his linking of the Riemann-Roch theorem with elliptic differential operators-the birth of the index theorem.

Atiyah's work on the related equations describing magnetic monopoles in $\mathbf{R}^{3}$ was rather different. These gaugetheoretic equations, the Bogomolny equations, were close cousins of the instantons but defined on $\mathbf{R}^{3}$. They describe static configurations, and Atiyah's engagement came from discussions with the mathematical physicist Nick Manton on the conjectural dynamics of slowly moving monopoles. Arguing that slow motion could be approximated by geodesic motion on the space of static solutions presented the question of finding the natural metric on the moduli space. When I came back to Oxford from an academic year in Stony Brook in 1984 I explained to him how both well-known and new hyperkähler metrics could be obtained by a quotient construction from flat space involving an adaptation of the theory of moment maps, but, spurred by his recall of the work with Bott on the YangMills equations on a Riemann surface, he insisted on treating the moduli space as an infinite-dimensional hyperkähler quotient and us working out the metric for two monopoles.

Working extensively that summer, I managed to use twistor theory to do this, but this was to Michael too complicated, so he derived the formula it gave by essentially elementary means, carefully marshalling known properties of the metric involving symmetry, spectral curves, and Donaldson's rational maps until the specific solution to 
the differential equation dropped out. We were then able to analytically describe some geodesics and discuss the scattering in detail, a project he pursued enthusiastically. He even convinced researchers at IBM's lab in the UK to test the power of their processors by producing a video of this phenomenon.

This ability to fashion proofs in an understandable form was key to the appeal of many of his papers and also to his lectures. In fact, the two topics above formed the basis of his series of Fermi Lectures on Yang-Mills theory [3] and the Porter Lectures on monopoles [4].

\section{References}

[1] Atiyah M, Lebrun C. Curvature, cones and characteristic numbers, Math. Proc. Cambridge Philos. Soc. 155 (2013), 13-37. MR3065256

[2] Atiyah M, Hitchin NJ, Singer IM. Deformations of instantons, Proc. Nat. Acad. Sci. USA 74 (1977), no. 7, 26622663. MR0458424

[3] Geometry of Yang-Mills Fields, Lezioni Fermiane, Accademia Nazionale dei Lincei \& Scuola Normale Superiore, Pisa, 1979.

[4] Atiyah M, Hitchin NJ. The geometry and dynamics of magnetic monopoles, M. B. Porter Lectures, Princeton University Press, Princeton, NJ, 1988. MR934202

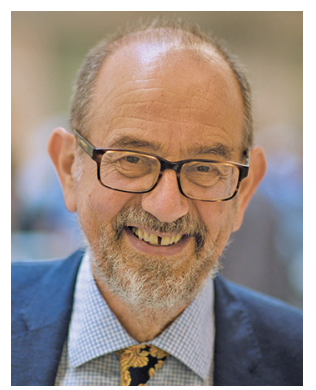

Nigel Hitchin

\section{Michael Atiyah and Physics}

\section{Edward Witten}

Michael Atiyah played a major role, starting in the mid1970s, in redefining the relationship between mathematics and physics.

By that time, theoretical physics had reached a major turning point with the emergence of the Standard Model of particle physics, based on nonabelian gauge theory or Yang-Mills theory. In a sense, theory had caught up with experiment, though it took a while for that to be clear. The

Edward Witten is a professor in the School of Natural Sciences at the Institute for Advanced Study, Princeton. His email address is wi tten@ias.edu. decades-long process that led to the Standard Model had been largely driven by experiment and by considerations of quantum theory that were rather far afield from the vantage point of most mathematicians. Conversely, during this period the ideas of modern mathematics seemed largely irrelevant to physicists grappling with elementary particles. A physics graduate student of the period, for example, would most probably never hear about a homology group, let alone something more contemporary like the Atiyah-Singer index theorem.

By the mid-1970s, the gauge theory revolution had created for physicists a new situation that would call for greater mathematical sophistication. But this was understood only gradually. Michael Atiyah and other mathematicians who became interested in what physicists were doing in quantum gauge theory played an important role in the process.

An early turning point came in 1976. A puzzle about the Standard Model known as the $\mathrm{U}(1)$ problem, identified by Murray Gell-Mann and Steve Weinberg, among others, was abruptly solved by Gerard 't Hooft by studying the Dirac equation in the field of a gauge theory instanton. Soon, Albert Schwarz showed that the key facts were best understood in the context of the Atiyah-Singer index theorem. Few physicists at the time knew what to make of this.

I first met Atiyah when he visited MIT in the spring of 1977, invited by Roman Jackiw. At the time, he was explaining his work with Richard Ward, solving the instanton equation on $\mathbb{R}^{4}$ by use of the Penrose twistor transform. His lectures had a big impact in the math and physics communities in the Cambridge (Massachusetts) area. Physicists at the time were very interested in solving the instanton equations because of speculation by Alexander Polyakov about the dynamics of gauge theories. However, the ingredients in the twistor transform of the instanton equation-complex manifolds, sheaf cohomology, fiber bundles - were quite unfamiliar to me and most other physicists.

By January 1978, when Atiyah invited me to visit Oxford for a few weeks, he was lecturing at the Maths Institute about a more precise understanding of instantons-the Atiyah-Drinfeld-Hitchin-Manin (ADHM) construction. I can well remember my perplexity in this period. Clearly, Atiyah and his colleagues were saying interesting and remarkable things about the nonlinear classical equations of nonabelian gauge theory. At the same time, it was very hard to imagine how their results could be applied to the questions of quantum dynamics that most interested physicists. Physical applications of the ADHM construction seemed far away.

Toward the end of my visit, Atiyah showed me two 
physics papers that I had not seen before. By this time, it was known that nonabelian gauge theories can describe magnetic monopoles as well as ordinary elementary particles. Ordinary particles arise by quantization of fields and are in representations of the gauge group $G$; monopoles are classified by topology. But Peter Goddard, Jean Nuyts, and David Olive (GNO) had suggested that magnetic charge can be interpreted as a weight of a "dual gauge group" $G^{\vee}$, and Olive with Claus Montonen had gone on to suggest a quantum duality between theories with gauge groups $G$ and $G^{\vee}$. Atiyah told me that the GNO dual gauge group was the same as the dual group introduced by Robert Langlands in the Langlands program and that he thought there was something very deep here. He urged me to go to London to discuss the matter with Olive.

By the time I got to London, I was skeptical. Like other physicists of the time, I had never heard of the Langlands program and I had no idea what to make of Atiyah's observation that the Langlands and GNO dual groups were the same. But I could see that technically the Montonen-Olive proposal was not correct if taken literally. However, by the end of the day, Olive and I understood that the technical objections to Montonen-Olive duality are absent in the supersymmetric case, and we had formulated a number of ideas that eventually (in the mid-1990s) were important in understanding it more fully.

Atiyah's deeper idea-that the Langlands program should somehow be tied up with electric-magnetic duality in four-dimensional gauge theory-remained in limbo for much longer. A concrete understanding depended on many intervening developments in both math and physics and only emerged in the mid-2000s. The full scope of this relationship is probably still far out of sight.

I will mention just a few highlights of the following decade. At the 1979 Cargése summer school, Atiyah and Raoul Bott undertook to educate physicists about Morse theory. I and most (or all?) of the physicists there had certainly never been exposed to Morse theory before. Another highlight was a conference in Texas where Atiyah and Is Singer began to elucidate the topological meaning of what physicists know as perturbative anomalies in gauge theory. This helped introduce physicists to a deeper understanding of fermion path integrals. Two papers by Atiyah and Bott in these years were ultimately influential for physicists. Their 1983 paper "The Yang-Mills equations over Riemann surfaces" introduced ideas that were important later in understanding quantum gauge theories in two dimensions. Their 1984 paper "The moment map and equivariant cohomology" helped lead to the important technique of "localization" in supersymmetric quantum field theory. Starting in the mid-1980s, the emergence of string theory greatly widened the horizons of physicists and ex- panded the scope of interaction between physicists and mathematicians. Among many other things, this led to unexpected applications of the ADHM construction in physics. Unfortunately, to explain all that here would take us too far afield.

In 1987, Atiyah twice visited the Institute for Advanced Study, and he was more excited than I could remember. What he was excited about was Floer theory (of symplectic manifolds or of flat connections on a three-manifold) which he thought should be interpreted as the Hamiltonian formulation of a quantum field theory. This quantum field theory was supposed to be, in language that was introduced later, a topological quantum field theory, which would be related to Gromov invariants of a symplectic manifold or Donaldson invariants of a four-manifold. The idea of topological quantum field theory was mostly Atiyah's conception. Atiyah set for me the task of trying to interpret what he was saying in the language of physicists. At first, this was difficult, for a variety of technical reasons. For example, the fermionic symmetry used by Floer had spin 0 , as opposed to the half-integral spin of spacetime supersymmetries as studied by physicists. But eventually I realized that a simple "twisting" of supersymmetric field theories could give a theory with the properties that Atiyah wanted. This gave, at a formal level, a reformulation of the Gromov and Donaldson invariants in a language that was natural to physicists.

The other problem that Atiyah recommended for physicists in the years 1987-8 was to understand the Jones polynomial of a knot via quantum field theory. I had never heard of the Jones polynomial before Atiyah recommended this problem, and this certainly put me in the majority among physicists. The challenge about the Jones polynomial that Atiyah posed was specifically to find a description of it with manifest topological invariance. By 1987-8, a number of constructions of the Jones polynomial were known, but topological invariance was never manifest a priori; it was always proved by checking generators and relations.

The following year brought many new clues about the Jones polynomial in work by, among others, Erik Verlinde, Greg Moore and Nathan Seiberg, and Akihiro Tsuchiya and Yukihiro Kanie. Eventually, at a meeting in Swansea, where I had the benefit of further discussions with Atiyah and with Graeme Segal, I had the good fortune to put some of the pieces together and interpret the Jones polynomial in terms of a three-dimensional gauge theory with the Chern-Simons function as its action.

This answered some of the questions, but actually Atiyah's vision about the Jones polynomial had two important aspects that were vindicated only long afterwards. First, Atiyah predicted that the argument $q$ of the Jones 
polynomial should ultimately be understood as a parameter that counts instantons on a four-manifold. In 1988-9, this idea looked to me like a bridge too far, but something along these lines was actually understood in the last decade. Various things were needed first, including the invention of a refinement of the Jones polynomial, known as Khovanov homology, and a greatly enriched understanding by physicists of the consequences of electricmagnetic duality. Also, Atiyah advocated that a natural explanation of the three-dimensional invariance of the Jones polynomial should have an extension to explain the spectral parameter of integrable systems and the associated Yang-Baxter equation. Something along these lines was understood only in the last few years in the work of Kevin Costello.

Actually, one facet of Atiyah's vision is still unclear as of 2019. Atiyah was always extremely interested in the spectral parameter that appears in the twistor transform of instantons-as in the ADHM construction-and in the construction of monopoles-as explored in a book that he wrote with Nigel Hitchin. He often expressed a suspicion that the monopole spectral parameter should be related to the spectral parameter of integrable spin systems and integrable models of lattice statistical mechanics. As of this writing, there is hope that something along these lines will emerge in further developments from the work of Costello.

Going back to Donaldson theory, after formulating it in terms of a twisted version of supersymmetric gauge theory, I thought that this would lead to immediate progress, but that was not the case. In the period around 1990, Atiyah probably understood better than I did the following essential point: to contribute something new to Donaldson theory, physicists would need some sort of strong coupling methods, since anything that could be said for weak coupling would involve retracing the steps that mathematicians had already taken. Eventually, Seiberg and I were able to apply strong coupling methods to this problem, leading to a relationship between Donaldson theory and an abelian theory with monopoles (Seiberg-Witten theory).

Michael Atiyah worked with physicists on many occasions. I will describe the background to one of the papers he wrote with physicists, "An M-theory flop as a large $N$ duality," written in the year 2000 with Juan Maldacena and Cumrun Vafa. Early in his career, Atiyah had explored the "small resolutions" of certain complex threefold singularities. By the mid-1990s, it was known that these small resolutions are important in the physics of Calabi-Yau manifolds, and it was also understood that nonperturbative "dualities" can relate string theory on a Calabi-Yau threefold to M-theory on a manifold of $G_{2}$ holonomy.
Putting these two lines of thought together, Atiyah, Maldacena, and Vafa explored the significance in M-theory of isolated singularities of $G_{2}$ manifolds and their resolutions. This was new at the time but is now regarded as an important direction. In the spring of 2001, Atiyah spent several months at Caltech, where I was on sabbatical. We had a memorable collaboration on this topic, leading to our paper "M-theory dynamics on a manifold of $G_{2}$ holonomy."

Atiyah, along with colleagues such as Raoul Bott and Is Singer, played an enormous role in introducing new ideas and encouraging and teaching physicists to study quantum field theory from new points of view. It took many twists and turns for these lessons to be really learned and absorbed in the physics world. Atiyah always believed that the study of quantum field theory as a tool in geometry had to be integrated with the study of more "physical" aspects of quantum field theory. His vision and clairvoyance have had a truly far-reaching influence.

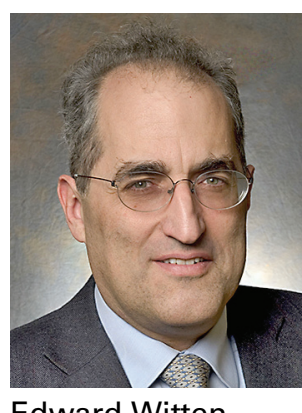

Edward Witten

\section{Recollections of Michael Atiyah}

\section{Simon Donaldson}

My main interaction with Michael Atiyah fits neatly into the decade of the 1980s, from the time when I arrived in Oxford in 1980 as a new graduate student until his departure in 1990 to take up the Mastership of Trinity College, Cambridge. While we perhaps did not perceive so at the time, it was an extraordinary decade. There were two large groups in the Mathematical Institute in Oxford, one led by Atiyah and the other by Penrose. These had come together, a few years before, through the Penrose "twistor" construction that had brought ideas from complex geometry into mathematical physics. Atiyah's interests centered on Yang-Mills theory, a theme that he developed in

Simon Donaldson is a permanent member at the Simons Center for Geometry and Physics in Stony Brook and a professor at Imperial College London. His email address is s.donaldson@ic.ac.uk. 
many directions. One prominent achievement was the Atiyah-Drinfeld-Hitchin-Manin (1978) description of all finite-energy solutions of the Yang-Mills instanton equation on $\mathbf{R}^{4}$, built on the twistorial construction that translates the problem into one about holomorphic vector bundles over $\mathrm{CP}^{3}$, thus connecting Yang-Mills theory to algebraic geometry. Another 1978 paper of Atiyah, Hitchin, and Singer took a more differential geometric direction, setting up the foundations for the theory of self-dual Riemannian four-manifolds and the Yang-Mills instantons over them. A third direction brought in topology: this was represented by the 1982 paper of Atiyah and Bott on the Yang-Mills equations over Riemann surfaces and a 1978 paper of Atiyah and Jones proposing a stabilization conjecture for the homology of instanton moduli spaces, partly motivated by an analogy with results of Segal on spaces of rational maps.

Over that decade, Atiyah's interests and activity moved over a variety of other topics. One of his long-standing interests was in the Bogomolny equation for monopoles on $\mathbf{R}^{3}$; others involved moment maps for group actions in symplectic geometry and equivariant cohomology, developing in part from the Atiyah-Bott paper mentioned above. One exciting application of localization techniques came in the influential proposal of Witten to prove the Atiyah-Singer index theorem by applying (formally) localization in the loop space of a manifold. Later in the decade the Jones knot invariant and the general notion of a topological field theory came into prominence (the latter influenced particularly by ideas of Segal). The close of the decade saw the arrival of "mirror symmetry" in geometry. One of the highlights of each year was the June trip to Bonn for the Arbeitstagung, where by tradition Atiyah gave the opening lecture. His last lecture in this series (circa 1990) discussed a paper of Candelas et al. involving mirror symmetry for Calabi-Yau hypersurfaces in weighted projective space.

Some of my most vivid memories from that time come from the wonderful seminars given by Atiyah, usually leading off his Monday afternoon seminar series with the first seminar of the term. The topics moved over many fields. In one example he discussed classical results on determinantal representations of plane curves (i.e., equations $\left.\operatorname{det}\left(A_{0} Z_{0}+A_{1} Z_{1}+A_{2} Z_{2}\right)=0\right)$ in connection with Hitchin's work from the early 1980 s on Nahm's equation and spectral curves. In another lecture the topic was a connection between the Dedekind $\eta$-function and the $\eta$ invariant of Atiyah, Patodi, and Singer, emerging from work of Witten, Quillen, Bismut, Freed, and others in the mid-1980s. Another memorable seminar was based on Atiyah's paper "Convexity and commuting Hamiltonians." This began at a down-to-level level with Horn's inequali- ties for the eigenvalues of a Hermitian matrix, which were soon embedded in symplectic geometry and a general theory of convexity results for moment maps. The common feature of all these talks was that they were immensely enjoyable experiences for the audience, full of Atiyah's excitement, drawing together old and new mathematics in surprising ways.

Atiyah occupied a large office with a fine view surveying the area of Oxford around St. Giles church. In his company one felt that one likewise surveyed with him the mathematical scene, and he would pour out a continual stream of ideas, suggestions, and observations. The fact that he travelled widely and talked to everybody meant that he always had many strands of current thought in his hands, which he would constantly weave into new patterns, seeking connections between different developments. Just as one example of a suggestion that was important to me, I remember him telling me about a variational point of view on symplectic quotients that he had heard about during a visit to Harvard. He suggested that perhaps there was some similar functional in the problem of the existence of Hermitian Yang-Mills connections on stable vector bundles. With this prompt it was not hard to find the functional, which, with a number of variants, has been important in complex differential geometry.

I met Michael regularly after 1990, and he remained an inspiration and source of wise advice and support in countless ways. His 2001 paper with Witten is an important reference for those of us attempting to understand sevendimensional manifolds with exceptional holonomy. I last saw him in August 2018 at the ICM in Rio. He was frail but otherwise as ever, overflowing with ideas and enthusiasm for mathematics.

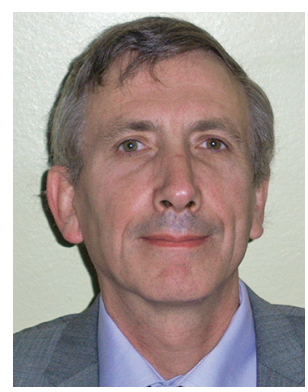

Simon Donaldson 


\section{Moment Maps and Convexity: Memories of Michael Atiyah}

\section{Frances Kirwan}

In the early 1980s, when I was Michael Atiyah's student, moment maps, convexity, and their links with each other and with algebraic and differential geometry and mathematical physics formed an important focus of his interests [2-5, 7-9].

I first met Michael around Christmas 1980 when I was in my final year as a Cambridge undergraduate wondering what to do next and whether I should apply for PhD places. I had summoned up the courage to look up his home phone number in the Oxford telephone directory and ring him. I was horribly embarassed when he answered the phone sounding very sleepy, although it was close to lunch time, and I discovered that he had just returned from the United States and I had woken him up. Nonetheless he was very friendly and set up a meeting with me at which he gave me much helpful advice and told me to mention his name on my application if I decided to apply to Oxford. I did that and had the huge good fortune to end up in the Mathematical Institute nine months later, joining other students in the geometry group, including Simon Donaldson, John Roe (who very tragically died a year before his former supervisor), Michael Murray, and Jacques Hurtubise.

Nobody who ever met Michael Atiyah will be surprised to be told that he was a wonderful supervisor to have: always full of an exciting mix of ideas and enthusiasm. He also had around him at the time a fantastic group of colleagues and visitors as well as students. Raoul and Phyllis Bott spent the academic year 1981-2 in Oxford, and Cliff Taubes (who had recently completed his PhD) also visited. Michael and Raoul were writing their fundamental papers on the Yang-Mills equations over a Riemann surface [7] (submitted March 1982, based on a preliminary account [6] written in 1980) and on the moment map and equivariant cohomology [8] (submitted December 1982). Michael had just completed his paper "Convexity and commuting Hamiltonians" [2] (submitted March 1981); he had recently learnt that Victor Guillemin and Shlomo Sternberg had discovered similar results independently and essentially simultaneously [14]. He was also intrigued by the observation (made to him by Sternberg and David Mumford) of a relationship between moment maps

Frances Kirwan is the Savilian Professor of Geometry at the University of Oxford. Her email address is ki rwan@maths.ox.ac.uk. and Mumford's geometric invariant theory (GIT). I was able to hear about all these ideas from Michael as soon as I started as his student, and he immediately gave me a beautiful problem to work on: to explore whether the same sorts of results he and Raoul had found for the Yang-Mills functional in an infinite-dimensional setting also hold for the normsquare of a moment map in the situation of a Hamiltonian action of a compact group $K$ on a compact symplectic manifold $M$, suggesting that his convexity results for torus actions might be relevant to this, as indeed they turned out to be.

A moment map for a Lie group $G$ acting on a symplectic manifold $(X, \omega)$ is a smooth map $\mu: X \rightarrow \mathfrak{g}^{*}$ that is $G$-equivariant with respect to the coadjoint action of $G$ on the dual of its Lie algebra $g$ and is such that the component $\mu \cdot a: X \rightarrow \mathbb{R}$ of $\mu$ along any $a \in \mathfrak{g}$ is a Hamiltonian function for the infinitesimal action of $a$ on $X$ (that is, the 1 -form $d(\mu \cdot a)$ corresponds under the duality defined by $\omega$ to the vector field on $X$ determined by $a$ ). The restriction of the symplectic form $\omega$ to $\mu^{-1}(0)$ is degenerate precisely along the orbits of $G$, and the "symplectic quotient" $\mu^{-1}(0) / G$ inherits a (stratified) symplectic structure.

An infinite-dimensional moment map plays a crucial role in the Atiyah-Bott paper [7] on the Yang-Mills equations over a Riemann surface, which was motivated by theoretical physics and brings together a wide range of different mathematical topics, including algebraic topology and Morse theory, algebraic geometry and number theory, gauge theory and analysis. The main object of study is the moduli space $M_{\Sigma}(n, d)$ of semistable holomorphic vector bundles of rank $n$ and degree $d$ over a compact Riemann surface $\Sigma$ of genus $g \geqslant 2$ (and more generally moduli spaces of principal $G$-bundles for a compact Lie group $G$ ). Here a holomorphic vector bundle $E$ over $\Sigma$ is (semi)stable if it has no proper subbundle $F$ with slope $\boldsymbol{\mu}(F):=$ degree $(F) / \operatorname{rank}(F)$ (strictly) greater than the slope $\boldsymbol{\mu}(E)$ $=d / n$ of $E$. Any semistable bundle $E$ has a JordanHölder filtration

$$
0=E_{0} \subset E_{1} \subset \cdots \subset E_{m}=E
$$

by subbundles of the same slope such that each subquotient $E_{j} / E_{j-1}$ is stable for $1 \leqslant j \leqslant m$, and the associated graded bundle

$$
\operatorname{gr}(E)=\bigoplus_{j=1}^{m} E_{j} / E_{j-1}
$$

is independent up to isomorphism of the choice of filtration. Two semistable bundles $E$ and $E^{\prime}$ over $\sum$ of rank $n$ and degree $d$ are said to be $S$-equivalent (after Seshadri) and represent the same point in $M_{\Sigma}(n, d)$ if and only if $\operatorname{gr}(E) \cong \operatorname{gr}\left(E^{\prime}\right)$. When $n$ and $d$ are coprime then semistability is equivalent to stability and $M_{\Sigma}(n, d)$ is the moduli 
space of stable rank $n$ and degree $d$ bundles over $\Sigma$ up to isomorphism.

There are many different ways to think about the geometry of the moduli space $M_{\Sigma}(n, d)$. It is a complex projective variety of dimension $n^{2}(g-1)+1$, and when $n$ and $d$ are coprime it is a compact Kähler manifold. It can be constructed as an infinite-dimensional analogue of a quotient in the sense of Mumford's geometric invariant theory of an infinite-dimensional complex affine space $C(n, d)$ of unitary connections on a fixed $C^{\infty}$ hermitian vector bundle $\mathscr{\varphi}_{0}$ of rank $n$ and degree $d$ by the action of the complexified gauge group $\mathcal{G}_{\mathbb{C}}(n, d)$ of complex automorphisms of $\mathscr{E}_{0}$. Atiyah and Bott observed that this infinite-dimensional affine space $C(n, d)$ has a natural Kähler structure that is invariant under the gauge group $G(n, d)$ of unitary automorphisms of $\mathscr{E}_{0}$ and that associating to a connection its curvature can be regarded as a moment map for the action of $G(n, d)$ on $C(n, d)$. They showed that adding a suitable central constant determined by the topological invariants $n$ and $d$ gives a moment map

$$
\mu: C(n, d) \rightarrow \operatorname{Lie} G(n, d)^{*}
$$

whose corresponding symplectic quotient $\mu^{-1}(0) / \mathcal{G}(n, d)$ can be identified with the moduli space $M_{\Sigma}(n, d)$ via the theorem of Narasimhan and Seshadri relating (semi)stability of bundles to unitary representations of the fundamental group $\pi_{1}(\Sigma)$. The normsquare of this moment map is (up to the addition of a constant) the Yang-Mills functional for the group $G=U(n)$ over $\Sigma$, and its critical points are the solutions to the Yang-Mills equations whose analogues over Minkowski space and $\mathbb{R}^{4}$ were well known to be important in physics. The aim of [7] was to apply Morse theory to the Yang-Mills functional to show that it is equivariantly perfect (in the sense that it induces $G(n, d)$-equivariant Morse inequalities that are actually equalities) and to use this to study the cohomology of the quotient $M_{\Sigma}(n, d)$ of its minimum by the gauge group $\mathcal{G}(n, d)$.

In fact, serious analytic difficulties arise with this program, not only because of the infinite-dimensionality of the picture but also because the Yang-Mills functional is a long way from being a Morse function in the traditional sense, or even a Morse-Bott function, as in general the connected components of its critical locus have singularities. The required analysis showing that Yang-Mills paths of steepest descent converge appropriately to critical points was later carried out by Daskalopoulos [12], but Atiyah and Bott avoided its use by constructing directly the associated "Morse stratification" of the space $C(n, d)$. In complex dimension one the Newlander-Nirenberg integrability condition for $\bar{\partial}$-operators holds vacuously, so the space $C(n, d)$ of unitary connections of $\mathscr{E}_{0}$ can be identified with the space of holomorphic structures on $\mathscr{\wp}_{0}$, and thus $C(n, d)$ can be stratified using the HarderNarasimhan type of a holomorphic vector bundle defined as follows. Any holomorphic vector bundle $E$ has a canonical filtration (its Harder-Narasimhan filtration)

$$
0=E_{0} \subset E_{1} \subset \cdots \subset E_{s}=E
$$

such that each subquotient $E_{j} / E_{j-1}$ is semistable and their slopes $\boldsymbol{\mu}\left(E_{j} / E_{j-1}\right)$ are strictly decreasing for $1 \leqslant$ $j \leqslant s$. The Harder-Narasimhan type of $E$ is then determined by the ranks and degrees of the semistable bundles $E_{j} / E_{j-1}$. In order to describe how bundles of one HarderNarasimhan type can degenerate to another (using work of Shatz), Atiyah and Bott encode the type $\boldsymbol{\mu}$ of $E$ as the vector $\left(\mu_{1}, \ldots, \mu_{n}\right)=\left(d_{1} / n_{1}, \ldots, d_{s} / n_{s}\right)$ in which the slope $d_{j} / n_{j}$ of $E_{j} / E_{j-1}$ appears $n_{j}=\operatorname{dim}\left(E_{j} / E_{j-1}\right)$ successive times or, equivalently, as the convex polygon $P$ with vertices $(0,0),\left(\operatorname{rank} E_{1}\right.$, degree $\left.E_{1}\right), \ldots,\left(\operatorname{rank} E_{s}\right.$, degree $\left.E_{s}\right)=$ $(n, d)$. The subset $C_{\boldsymbol{\mu}}(n, d)$ of $C(n, d)$ consisting of holomorphic structures on $\mathscr{\varphi}_{0}$ of Harder-Narasimhan type $\boldsymbol{\mu}$ is a locally closed complex submanifold of $C(n, d)$ with finite codimension

$$
d_{\boldsymbol{\mu}}=\sum_{\mu_{i}>\mu_{j}}\left(\mu_{i}-\mu_{j}+g-1\right),
$$

and its closure is contained in the union of the strata labelled by $\boldsymbol{\mu}^{\prime}$ with $\boldsymbol{\mu}^{\prime} \geqslant \boldsymbol{\mu}$ in the sense that the polygon $P^{\prime}$ associated to $\boldsymbol{\mu}^{\prime}$ lies above the polygon $P$. In particular, when $\boldsymbol{\mu}=(d / n, \ldots, d / n)$ then $C_{\boldsymbol{\mu}}(n, d)=C^{s s}(n, d)$ is the open subset of semistable holomorphic structures on $\mathfrak{\wp}_{0}$.

An elegant argument (which has become known as the Atiyah-Bott lemma) shows that the Thom-Gysin long exact sequence

$$
\begin{aligned}
\cdots \rightarrow H_{G(n, d)}^{*-2 d_{\mu}} & \left(C_{\boldsymbol{\mu}}(n, d) ; \mathbb{Q}\right) \\
\rightarrow & H_{\mathcal{G}(n, d)}^{*}\left(C \backslash \bigcup_{\boldsymbol{\mu}^{\prime}>\boldsymbol{\mu}} C_{\boldsymbol{\mu}^{\prime}}(n, d) ; \mathbb{Q}\right) \\
& \rightarrow H_{\mathcal{G}(n, d)}^{*}\left(C \backslash \bigcup_{\boldsymbol{\mu}^{\prime} \geqslant \boldsymbol{\mu}} C_{\boldsymbol{\mu}^{\prime}}(n, d) ; \mathbb{Q}\right) \rightarrow \cdots
\end{aligned}
$$

breaks up into short exact sequences by proving that the composition of $H_{\mathcal{G}(n, d)}^{*-2 d_{\mu}}\left(C_{\boldsymbol{\mu}}(n, d) ; \mathbb{Q}\right) \rightarrow$ $H_{\mathcal{G}(n, d)}^{*}\left(C \backslash \cup_{\boldsymbol{\mu}^{\prime}>\boldsymbol{\mu}} C_{\boldsymbol{\mu}^{\prime}}(n, d) ; \mathbb{Q}\right)$ with the restriction map

$$
H_{\mathcal{G}(n, d)}^{*}\left(C \backslash \bigcup_{\boldsymbol{\mu}^{\prime}>\boldsymbol{\mu}} C_{\boldsymbol{\mu}^{\prime}}(n, d) ; \mathbb{Q}\right) \rightarrow H_{\mathcal{G}(n, d)}^{*}\left(C_{\boldsymbol{\mu}}(n, d) ; \mathbb{Q}\right)
$$

is injective. Indeed this composition is multiplication by the equivariant Euler class

$$
e_{\boldsymbol{\mu}} \in H_{G(n, d)}^{2 d_{\mu}}\left(C_{\boldsymbol{\mu}}(n, d) ; \mathbb{Q}\right)
$$

of the normal bundle to $C_{\mu}(n, d)$ in $C(n, d)$, and so it suffices to show that $e_{\mu}$ is not a zero divisor in 
$H_{\mathcal{G}(n, d)}^{*}\left(C_{\boldsymbol{\mu}}(n, d) ; \mathbb{Q}\right)$, which is isomorphic to

$$
\bigotimes_{j=1}^{s} H_{\mathcal{G}\left(n_{j}, d_{j}\right)}^{*}\left(C^{s s}(n, d) ; \mathbb{Q}\right),
$$

where $\boldsymbol{\mu}=\left(d_{1} / n_{1}, \ldots, d_{s} / n_{s}\right)$. Since the central subgroup $S^{1}$ of $G\left(n_{j}, d_{j}\right)$ given by multiplication by scalars acts trivially on $C^{s s}\left(n_{j}, d_{j}\right)$, we have

$$
\begin{aligned}
H_{\mathcal{G}\left(n_{j}, d_{j}\right)}^{*} & \left(C^{s s}(n, d) ; \mathbb{Q}\right) \\
& \cong H^{*}\left(B S^{1} ; \mathbb{Q}\right) \otimes H_{\overline{\mathcal{G}}\left(n_{j}, d_{j}\right)}^{*}\left(C^{s s}(n, d) ; \mathbb{Q}\right),
\end{aligned}
$$

where $\overline{\mathcal{G}}\left(n_{j}, d_{j}\right)=\mathcal{G}\left(n_{j}, d_{j}\right) / S^{1}$ and the rational cohomology $H^{*}\left(B S^{1} ; \mathbb{Q}\right)$ of the classifying space of the circle group $S^{1}$ is a polynomial ring $\mathbb{Q}\left[u_{j}\right]$ on one variable $u_{j}$ in degree 2. Atiyah and Bott show that the component of the equivariant Euler class $e_{\mu}$ in

$$
\bigotimes_{j=1}^{s} \mathbb{Q}\left[u_{j}\right] \otimes H_{\overline{\mathcal{G}}\left(n_{j}, d_{j}\right)}^{0}\left(C^{s s}(n, d) ; \mathbb{Q}\right) \cong \mathbb{Q}\left[u_{1}, \ldots, u_{s}\right]
$$

is a nonzero polynomial (the product of the weights of the torus $\left(S^{1}\right)^{S}$ on the normal bundle to $C_{\boldsymbol{\mu}}(n, d)$ in $\left.C(n, d)\right)$ and therefore that $e_{\mu}$ is not a zero divisor in $H_{\mathcal{G}(n, d)}^{*}\left(C_{\boldsymbol{\mu}}(n, d) ; \mathbb{Q}\right)$, as required. Removing each stratum $C_{\mu}(n, d)$ from $C(n, d)$ one by one, leaving an open subset of $C(n, d)$ each time, and using the Atiyah-Bott lemma give us the equivariant perfection of the stratification:

$$
\begin{aligned}
& \operatorname{dim} H_{\mathcal{G}(n, d)}^{i}(C(n, d) ; \mathbb{Q}) \\
& \quad=\sum_{\boldsymbol{\mu}} \operatorname{dim} H_{\mathcal{G}(n, d)}^{i-2 d_{\mu}}\left(C_{\boldsymbol{\mu}}(n, d) ; \mathbb{Q}\right),
\end{aligned}
$$

where

$$
H_{\mathcal{G}(n, d)}^{*}\left(C_{\boldsymbol{\mu}}(n, d) ; \mathbb{Q}\right) \cong \bigotimes_{j=1}^{s} H_{\mathcal{G}\left(n_{j}, d_{j}\right)}^{*}\left(C^{s s}(n, d) ; \mathbb{Q}\right) \text {. }
$$

Since $C(n, d)$ is an affine space and so homotopically trivial, we have

$$
H_{\mathcal{G}(n, d)}^{i}(C(n, d) ; \mathbb{Q}) \cong H^{i}(B G(n, d) ; \mathbb{Q}),
$$

which Atiyah and Bott showed has dimension given by the coefficient of $t^{i}$ in the Poincaré series

$$
\begin{aligned}
& P_{t}(B G(n, d)) \\
& \quad=\prod_{k=1}^{n}\left(1+t^{2 k-1}\right)^{2 g} /\left(1-t^{2 n}\right) \prod_{k=1}^{n-1}\left(1-t^{2 k}\right)^{2} .
\end{aligned}
$$

This gave them an inductive formula (later made explicit by Zagier) for calculating the equivariant Betti numbers $\operatorname{dim} H_{G(n, d)}^{i}\left(C^{s s}(n, d) ; \mathbb{Q}\right)$ for the semistable stratum $C^{s s}(n, d)$. When $n$ and $d$ are coprime then $C^{s s}(n, d)=$
$C^{s}(n, d)$, and the moduli space $M_{\Sigma}(n, d)$ can be identified with the quotient $C^{s s}(n, d) / \overline{\mathcal{G}}_{\mathbb{C}}(n, d)$ where $\overline{\mathcal{G}}_{\mathbb{C}}(n, d)=G_{\mathbb{C}}(n, d) / \mathbb{C}^{*}$ acts freely on $C^{s s}(n, d)$. This means that

$$
\begin{aligned}
H_{\mathcal{G}(n, d)}^{*}\left(C^{s s}(n, d) ; \mathbb{Q}\right) & \\
& \cong H^{*}\left(B S^{1} ; \mathbb{Q}\right) \otimes H^{*}\left(M_{\Sigma}(n, d) ; \mathbb{Q}\right),
\end{aligned}
$$

and we obtain a formula for the Betti numbers of the moduli space $M_{\Sigma}(n, d)$ of semistable holomorphic vector bundles of rank $n$ and degree $d$ over $\Sigma$. We also obtain multiplicative generators for its rational cohomology, since by equivariant perfection of the stratification the restriction map

$$
H_{\mathcal{G}(n, d)}^{*}(C(n, d) ; \mathbb{Q}) \rightarrow H_{\mathcal{G}(n, d)}^{*}\left(C^{s s}(n, d) ; \mathbb{Q}\right)
$$

is surjective, and $H_{\mathcal{G}(n, d)}^{*}(C(n, d) ; \mathbb{Q}) \cong H^{*}(B G(n, d) ; \mathbb{Q})$ has generators

$$
a_{r} \in H^{2 r}(B G(n, d) ; \mathbb{Q}), b_{r}^{j} \in H^{2 r-1}(B G(n, d) ; \mathbb{Q})
$$

for $1 \leqslant j \leqslant 2 g$ and $f_{r} \in H^{2 r-2}(B G(n, d) ; \mathbb{Q})$

given by decomposing the Chern classes of the universal bundle over $B G(n, d) \times \Sigma$ using the Künneth decomposition of $H^{*}(B G(n, d) \times \Sigma ; \mathbb{Q})$.

Equivalent formulas for the Betti numbers of $M_{\Sigma}(n, d)$ when $n$ and $d$ are coprime, had already been obtained by Harder-Narasimhan and Desale-Ramanan using arithmetic geometry and the Weil conjectures. Although the methods used looked very different in some ways, Atiyah and Bott observed that there was a formal correspondence between them. Both methods are based on the stratification by Harder-Narasimhan-type of the moduli stack $\mathcal{M}_{\Sigma}(n, d)=\left[C(n, d) / \mathcal{G}_{\mathbb{C}}(n, d)\right]$ of bundles over $\Sigma$ (cf. $[1,13,15])$, although Atiyah and Bott did not use the language of stacks, together with inductive descriptions of the unstable strata in terms of moduli of semistable bundles of smaller rank, and the fact that when $n$ and $d$ are coprime the Betti numbers of the moduli space $M_{\Sigma}(n, d)$ are given by the dimensions of the cohomology groups of the semistable stratum of this stack. The methods are different, however, in how they use the stratification to deduce a version of the inductive formula

$$
\begin{aligned}
& P_{t}\left(\left[C^{s s}(n, d) / G_{\mathbb{C}}(n, d)\right]\right)=P_{t}\left(\left[C(n, d) / G_{\mathbb{C}}(n, d)\right]\right) \\
- & \sum_{\substack{\mu=\left(d_{1} / n_{1}, \ldots, d_{s} / n_{s}\right) \\
\neq(d / n, \ldots d / n)}} t^{2 d_{\mu}} \prod_{j=1}^{s} P_{t}\left(\left[C^{s s}\left(n_{j}, d_{j}\right) / G_{\mathbb{C}}\left(n_{j}, d_{j}\right)\right]\right)
\end{aligned}
$$

for the Poincaré series of the moduli stack $\mathcal{M}_{\Sigma}^{S S}(n, d)=$ $\left[C^{s s}(n, d) / G_{\mathbb{C}}(n, d)\right]$ of semistable holomorphic bundles of rank $n$ and degree $d$ over $\Sigma$ and to calculate 
the crucial ingredient given by the Poincaré series $P_{t}\left(\left[C(n, d) / G_{\mathbb{C}}(n, d)\right]\right)$ of the moduli stack $\mathcal{M}_{\Sigma}(n, d)=$ $\left[C(n, d) / G_{\mathbb{C}}(n, d)\right]$ of all holomorphic bundles of rank $n$ and degree $d$ over $\Sigma$.

In the approach motivated by gauge theory, the AtiyahBott lemma gives the equivariant perfection of the Morse stratification of the Yang-Mills functional and thus the inductive formula (2), while algebraic topology is used to calculate the Poincaré series $P_{t}(B G(n, d))=$ $P_{t}\left(\left[C(n, d) / G_{\mathbb{C}}(n, d)\right]\right)$ of the classifying space of the gauge group. The earlier arithmetic approach was based on the Weil conjectures, which can be used to calculate the Betti numbers of nonsingular complex projective varieties (or more generally projective ind-varieties) by counting points in associated varieties defined over finite fields. From this viewpoint we need to assume (as we may by a small perturbation) that the compact Riemann surface $\Sigma$ is a nonsingular complex projective curve defined over the rationals, and indeed over $\mathbb{Z}$, with good reduction modulo $p$ for all but finitely many primes $p$. Let $C$ be a nonsingular projective curve over a finite field $\mathbb{F}_{q}$ of characteristic $p$. Using his theory of matrix divisor classes, Weil constructed a canonical bijection from the set of isomorphism classes of vector bundles on $C$ defined over $\mathbb{F}_{q}$ to a double coset space

$$
\mathfrak{E} \backslash \mathrm{GL}_{n}\left(\mathbb{A}_{K}\right) / \mathrm{GL}_{n}(K),
$$

where $K=\mathbb{F}_{q}(C)$ is the function field of $C$ and $\mathbb{A}_{K}$ is its adèle ring, while $\mathfrak{K}$ is a maximal compact subgroup of $\mathrm{GL}_{n}\left(\mathbb{A}_{K}\right)$. It is more convenient to fix determinants; replacing $\mathrm{GL}_{n}$ with $\mathrm{SL}_{n}$, the double coset space

$$
\mathfrak{K}_{\Lambda} \backslash \mathrm{SL}_{n}\left(\mathbb{A}_{K}\right) / \mathrm{SL}_{n}(K)
$$

is in bijection with the set $\operatorname{Bun}_{\mathrm{SL}_{n}}^{\Lambda}\left(\mathbb{F}_{q}\right)$ of isomorphism classes of bundles $E$ on $C$ equipped with an isomorphism $\operatorname{det} E \cong \Lambda$ defined over $\mathbb{F}_{q}$, where the maximal compact subgroup $\mathfrak{K}_{\Lambda}$ of $\mathrm{SL}_{n}\left(\mathbb{A}_{k}\right)$ depends on the line bundle $\Lambda$. Siegel's mass formula gives us

$$
\tau\left(\mathrm{SL}_{n}\right)=\operatorname{vol}\left(\mathfrak{K}_{\Lambda}\right) \sum_{\mathscr{\delta} \in \operatorname{Bun}_{\mathrm{SL}_{n}}^{\Lambda}\left(\mathbb{F}_{q}\right)} \frac{1}{|\operatorname{Aut}(\mathscr{\zeta})|},
$$

where the Tamagawa number $\tau\left(\mathrm{SL}_{n}\right)$ is the total measure of $\mathrm{SL}_{n}\left(\mathbb{A}_{K}\right) / \mathrm{SL}_{n}(K)$ with respect to a right invariant Haar measure on $\mathrm{SL}_{n}\left(\mathbb{A}_{K}\right)$ and $\operatorname{vol}\left(\mathfrak{K}_{\Lambda}\right)$ is calculated with respect to the same measure. We have

$$
\tau\left(\mathrm{SL}_{n}\right)=1 \text {, }
$$

while $\operatorname{vol}\left(\mathfrak{K}_{\Lambda}\right)$ is given in terms of the zeta function $Z_{C}$ of $C$ as

$$
\operatorname{vol}\left(\mathfrak{K}_{\Lambda}\right)=q^{-\left(n^{2}-1\right)(g-1)} / Z_{C}\left(q^{-2}\right) \cdots Z_{C}\left(q^{-n}\right) .
$$

Partitioning $\operatorname{Bun}_{\mathrm{SL}_{n}}^{\Lambda}\left(\mathbb{F}_{q}\right)$ by Harder-Narasimhan type into subsets $\operatorname{Bun}_{\mathrm{SL}_{n}}^{\Lambda, \mu}\left(\mathbb{F}_{q}\right)$ and combining Siegel's mass formula with (3) and (4) gives

$$
\begin{aligned}
q^{\left(n^{2}-1\right)(g-1)} Z_{C}\left(q^{-2}\right) \cdots Z_{C}\left(q^{-n}\right) & \\
& -\sum_{\mu \neq(d / n, \ldots, d / n)} \sum_{\substack{\Lambda \in \operatorname{Bun}_{\mathrm{SL}_{n}}\left(\mathbb{F}_{q}\right)\\
}} 1 /|\operatorname{Aut}(\mathscr{\wp})|
\end{aligned}
$$

as the number of isomorphism classes defined over $\mathbb{F}_{q}$ of semistable bundles on $C$ with rank $n$ and degree $d$. The observation of Atiyah and Bott that these inductive methods for obtaining the Betti numbers of $M_{\Sigma}(n, d)$ (when $n$ and $d$ are coprime) are parallel, with the Siegel mass formula (derived from the additivity of counting points over finite fields) playing the role of the equivariant perfection of the Yang-Mills functional (derived from the Atiyah-Bott lemma) and (3) playing the role of the contractibility of the infinite-dimensional affine space $C(n, d)$, has been explored further in the decades since (see e.g. $[1,13]$ and the references therein).

When I became Michael Atiyah's student he told me about these beautiful ideas and about his suspicion that a vital ingredient in the Yang-Mills picture was that the Yang-Mills functional can be regarded as the normsquare of a moment map for the action of the gauge group $G(n, d)$ on the space $C(n, d)$. His hope (which turned out to be correct) was that a similar picture should emerge in finitedimensional situations when a compact Lie group $K$ acts on a compact symplectic manifold $(X, \omega)$ with moment map $\mu: X \rightarrow \mathfrak{k}^{*}$ where $\mathfrak{k}=$ Lie $K$. The arithmetic aspects should apply (and a close link with Mumford's geometric invariant theory should exist) when $(X, \omega)$ is a nonsingular complex projective variety with Fubini-Study Kähler form associated to a very ample vector bundle $L$ on $X$ and the complexification $G=K_{\mathbb{C}}$ of $K$ acts linearly on $X$ with respect to $L$. An important piece of evidence for this was the appearance of convexity in the Yang-Mills picture, together with his recent paper on convexity and commuting Hamiltonians. In this he studied the case when $K=T \cong\left(S^{1}\right)^{r}$ is a compact torus acting on a compact connected symplectic manifold $X$ preserving the symplectic form $\omega$ and with a moment map $\mu_{T}: X \rightarrow t^{*}$ where $\mathfrak{t}=\mathrm{Lie} T$. He showed that $\mu_{T}(X)$ is a convex polytope, the convex hull in $\mathrm{t}^{*}$ of the finite set $\mu_{T}\left(X^{T}\right)$, where $X^{T}$ is the set of $T$-fixed points in $X$. If moreover $(X, \omega)$ is Kähler, then the action of $T$ extends to its complexification $T_{\mathbb{C}} \cong\left(\mathbb{C}^{*}\right)^{r}$, and for any $x \in X$ the image $\mu_{T}\left(\overline{T_{\mathbb{C}} X}\right)$ of the

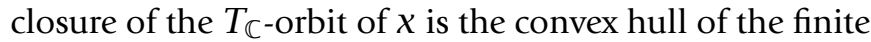
set $\mu_{T}\left(\overline{T_{\mathbb{C} X}} \cap X^{T}\right)$, with $\mu_{T}\left(\overline{T_{\mathbb{X} X}}\right)=\mu_{T}(X)$ for generic $x \in X$. The application of this to the maximal torus of an arbitrary compact Lie group with a Hamiltonian action on a compact connected symplectic manifold allows us to describe the Morse stratification of the normsquare of the moment map and show that it is equivariantly perfect by 
applying the Atiyah-Bott lemma, thus obtaining results similar to those of the Yang-Mills situation about the cohomology of the symplectic quotient. Michael Atiyah and Andrew Pressley also extended the convexity result in an infinite-dimensional setting involving the loop group of $K$ [9].

Moment maps and equivariant cohomology provide the title of another fundamental paper [8] by Atiyah and Bott written around the same time as the Yang-Mills paper [7]. This is closely related to independent work of Berline and Vergne [10] and was motivated by the DuistermaatHeckman formula for the pushforward by a moment map $\mu_{T}: X \rightarrow t^{*}$ for a torus action on a compact connected symplectic manifold $(X, \omega)$ of the symplectic measure given by $\omega^{n} / n$ !. This pushforward is always piecewise polynomial. Equivalently the stationary phase approximation for its Fourier transform is exact, so that

$$
\int_{X} e^{-i\left\langle\xi, \mu_{T}\right\rangle} \omega^{n} / n !=\sum_{F \in \mathcal{F}} e^{-i\left\langle\xi, \mu_{T}(F)\right\rangle} \int_{F} \frac{\omega^{\operatorname{dim} F}}{(\operatorname{dim} F) ! e_{F}(i \xi)}
$$

for any $\xi \in \mathfrak{t}$, where $\langle$,$\rangle is the canonical pairing between$ $t$ and $t^{*}$, while $\mathcal{F}$ is the set of connected components of the fixed point set $X^{T}$ for the $T$-action on $X$ (so that $\left.\mu_{T}\right|_{F}$ is constant for each $F \in \mathcal{F}$ ) and $e_{F}$ is the equivariant Euler class for the normal bundle to $F$ in $X$. If $F \in \mathcal{F}$, then $T \cong\left(S^{1}\right)^{r}$ acts trivially on $F$, and so there is a canonical isomorphism

$$
H_{T}^{*}(F ; \mathbb{C}) \cong H^{*}(F ; \mathbb{C}) \otimes H^{*}(B T ; \mathbb{C}),
$$

where $H^{*}(B T ; \mathbb{C})=\operatorname{Sym}\left(\mathfrak{t}_{\mathbb{C}}^{*}\right) \cong \mathbb{C}\left[u_{1}, \ldots, u_{r}\right]$ is a polynomial ring on $r$ generators of degree 2 . As in the proof of the Atiyah-Bott lemma, the component of $e_{F} \in H_{T}^{*}(F ; \mathbb{C})$ in

$$
H^{0}(F ; \mathbb{C}) \otimes H^{*}(B T ; \mathbb{C}) \cong \mathbb{C}\left[u_{1}, \ldots, u_{r}\right]
$$

is nonzero, and hence $e_{F}$ is not a zero divisor in $H_{T}^{*}(F ; \mathbb{C})$, because terms of positive degree in $H^{*}(F)$ are nilpotent. Thus $e_{F}$ is invertible in the localization $H^{*}(F ; \mathbb{C}) \otimes$ $\mathbb{C}\left(u_{1}, \ldots, u_{r}\right)$ of $H_{T}^{*}(F ; \mathbb{C})$. This enables us to interpret $\int_{F} \omega^{\operatorname{dim} F} /(\operatorname{dim} F) ! e_{F}(i \xi)$ as a rational function on $t$ and make sense of the identity (5).

Atiyah and Bott observed that the formula (5) can be interpreted as a consequence of well-known localization formulas in equivariant cohomology. For a compact group $K$ acting on a manifold $X$ they describe a de Rham version of equivariant cohomology as the basic complex $\Omega_{\mathfrak{k}}^{*}(X)$ of $\Omega^{*}(X) \otimes W(\mathfrak{k})$, where $\Omega^{*}(X)$ is the usual de Rham complex, $W(\mathfrak{k})=\Lambda \mathfrak{k}^{*} \otimes$ Sym $\mathfrak{k}^{*}$ is the Weil algebra, and elements $\phi$ of $\Omega^{*}(X) \otimes W(\mathfrak{k})$ are basic if $\iota(a) \phi=0$ and $\mathcal{L}(a) \phi=0$ for all $a \in \mathfrak{k}$. Here $W(\mathfrak{k})$ is graded by giving degree 1 to elements $\theta \in \mathfrak{k}^{*}$ in $\Lambda \mathfrak{k}^{*}$ and degree 2 to $u \in \mathfrak{k}^{*}$ regarded as in Sym $\mathfrak{k}^{*}$. Then $W(\mathfrak{k})$ is freely generated as a commutative graded algebra by a basis $\left\{\theta^{\alpha}\right\}$ of $\mathfrak{k}^{*}$ in degree 1 and the same basis denoted $\left\{u_{\alpha}\right\}$ regarded as in degree 2 . There is a differential operator $D$ on $W(\mathfrak{k})$ defined on the generators $\left\{\theta^{\alpha}\right\}$ and $\left\{u_{\alpha}\right\}$ in terms of the structure constants for the Lie algebra $k$. When $X$ is a point, $\Omega_{\mathfrak{k}}^{*}(X)$ is the basic subcomplex of $W(\mathfrak{k})$, which is given by polynomials on $k$ invariant under the adjoint action. A symplectic form $\omega \in \Omega^{2}(X)$ on $X$ is closed but is not in general equivariantly closed for a compatible group action on $X$. However, when a compact torus $T=\left(S^{1}\right)^{r}$ acts on $(X, \omega)$ with moment map $\mu_{T}: X \rightarrow \mathfrak{t}^{*}$ then $D\left(\omega-\mu_{T}\right)=0$, and so $\omega-\mu_{T}$ represents an equivariant cohomology class.

Atiyah and Bott consider $H_{T}^{*}(X ; \mathbb{C})$ as a module over $H^{*}(B T ; \mathbb{C})=\mathbb{C}\left[u_{1}, \ldots, u_{r}\right]$ and show that the kernel and cokernel of the restriction map

$$
i^{*}: H_{T}^{*}(X ; \mathbb{C}) \rightarrow \bigoplus_{F \in \mathcal{F}} H_{T}^{*}(F ; \mathbb{C})
$$

(with components $i_{F}^{*}$ for $F \in \mathcal{F}$ ) and the pushforward map

$$
i_{*}: \bigoplus_{F \in \mathcal{F}} H_{T}^{*}(F ; \mathbb{C}) \rightarrow H_{T}^{*}(X ; \mathbb{C})
$$

(with components $i_{*}^{F}$ for $F \in \mathcal{F}$ ) are torsion modules over $H^{*}(B T ; \mathbb{C})$, so their composition $i^{*} i_{*}$ is an isomorphism modulo torsion. Indeed $i^{*} i_{*}$ is given on $H_{T}^{*}(F ; \mathbb{C})$ for $F \in \mathcal{F}$ by multiplication by the equivariant Euler class $e_{F}$ of the normal bundle to $F$ in $X$, and the component of $e_{F}$ in

$$
H^{0}(F ; \mathbb{C}) \otimes H^{*}(B T ; \mathbb{C}) \cong \mathbb{C}\left[u_{1}, \ldots, u_{r}\right]
$$

is

$$
e_{F, 0}=\prod_{j=1}^{\operatorname{codim}_{\mathbb{R}}(F) / 2}\left(a_{j 1} u_{1}+\cdots+a_{j r} u_{r}\right),
$$

where the representation of $T=\left(S^{1}\right)^{r}$ on the normal to $F$ in $X$ at any point of $F$ has weights given by $a_{j 1} u_{1}+$ $\cdots+a_{j r} u_{r}$ for $1 \leqslant j \leqslant \operatorname{codim}_{\mathbb{R}}(F) / 2$. If we localize with respect to the nonzero polynomial $\prod_{F \in \mathcal{F}} e_{F, 0}$ in $\mathbb{C}\left[u_{1}, \ldots u_{r}\right]$, then it follows that

$$
\sum_{F \in \mathcal{F}} i_{F}^{*} / e_{F}
$$

is inverse to $i_{*}$, and that if $\phi \in H_{T}^{*}(X ; \mathbb{C})$, then

$$
\phi=\sum_{F \in \mathcal{F}} i_{*}^{F} i_{F}^{*} \phi / e_{F} .
$$

Pushing forward to a point and replacing $\phi$ with $\phi e^{\bar{\omega}}$ where $\bar{\omega}=\omega-\mu_{T}$ gives us the integration formula

$$
\int_{X} \phi e^{\bar{\omega}}=\sum_{F \in \mathcal{F}} \int_{F} i_{F}^{*}\left(\phi e^{\bar{\omega}}\right) / e_{F}
$$


with the Duistermaat-Heckman formula as the special case when $\phi=1$.

Atiyah and Bott also related these ideas to (the second half of) the paper [18] of Witten, who later produced a nonabelian version of localization using the normsquare $\|\mu\|^{2}$ of a moment map for a Hamiltonian action of a compact group $K$ on $X$ to study the integrals

$$
\int_{\mu^{-1}(0) / K}\left(\eta e^{\bar{\omega}}\right)_{0}
$$

over a symplectic quotient $\mu^{-1}(0) / K$ by localizing near the critical points of $\|\mu\|^{2}$. Here we assume that 0 is a regular value of $\mu$, and if $\alpha \in H_{K}^{*}(X ; \mathbb{C})$, then $\alpha_{0} \in$ $H^{*}\left(\mu^{-1}(0) / K ; \mathbb{C}\right) \cong H_{K}^{*}\left(\mu^{-1}(0) ; \mathbb{C}\right)$ is its image under the restriction map $H_{K}^{*}(X ; \mathbb{C}) \rightarrow H_{K}^{*}\left(\mu^{-1}(0) ; \mathbb{C}\right)$. Witten used these ideas applied to the Yang-Mills functional to find formulas for the evaluation on the moduli space $M_{\Sigma}(n, d)$ of any product of the generators

$$
\begin{gathered}
a_{r} \in H^{2 r}\left(M_{\Sigma}(n, d) ; \mathbb{C}\right), b_{r}^{j} \in H^{2 r-1}\left(M_{\Sigma}(n, d) ; \mathbb{C}\right) \\
\quad \text { for } 1 \leqslant j \leqslant 2 g \text { and } f_{r} \in H^{2 r-2}\left(M_{\Sigma}(n, d) ; \mathbb{C}\right)
\end{gathered}
$$

for $H^{*}\left(M_{\Sigma}(n, d) ; \mathbb{C}\right)$ as at $(1)$ above, and thus to extend Atiyah and Bott's description of the cohomology of $M_{\Sigma}(n, d)$ to include a complete set of relations among these generators (cf. $[16,17,19])$.

\section{References}

[1] Asok A, Doran B, Kirwan F. Yang-Mills theory and Tamagawa numbers, Bull. London Math. Soc. 40 (2008), 533567. MR2438072

[2] Atiyah M. Convexity and commuting Hamiltonians, Bull. London Math. Soc. 14 (1982), 1-15. MR642416

[3] Atiyah M. Gauge theory and algebraic geometry, Proc. Beijing Symposium on differential geometry and partial differential equations, Science Press, China, 1982, 1-20.

[4] Atiyah M. Angular momentum, convex polyhedra and algebraic geometry, Proc. Edin. Math. Soc. 26 (1983), 121-38. MR705256

[5] Atiyah M. The moment map in symplectic geometry, Global Riemannian Geometry, Horwood, 1984, 43-51.

\section{MR757204}

[6] Atiyah M, Bott R. Yang-Mills and bundles over algebraic curves, Proc. Indian Acad. Sci. 90 (1981), 11-20. MR653942

[7] Atiyah M, Bott R. The Yang-Mills equations over Riemann surfaces, Phil. Trans. Royal Soc. Series A 308 (1983), 523615 MR702806

[8] Atiyah M, Bott R. The moment map and equivariant cohomology, Topology 23 (1984), 1-28. MR721448

[9] Atiyah M, Pressley A. Convexity and loop groups, Prog. Math. 36 (1983), 33-64. MR717605

[10] Berline N, Vergne M. Classes caracteristiques equivariantes, formule de localisation en cohomologie equivariante, C. R. Acad. Sci. Paris Sér. I Math. 295 (1982), 539-541. MR685019
[11] Bott R. Lectures on Morse theory, old and new, Bull. Amer. Math. Soc. (NS) 7 (1982), 331-358 MR663786

[12] Daskalopoulos G. The topology of the space of stable bundles on a Riemann surface, J. Diff. Geom. 36 (1992), 699-746. MR1189501

[13] Gaitsgory D, Lurie J. Weil's conjecture for function fields, Annals of Math. Studies, 199, Princeton Univ. Press, 2019. MR3887650

[14] Guillemin V, Sternberg S. Convexity properties of the moment mapping, Invent. Math. 67 (1982), 491-513. MR664117

[15] Halpern-Leistner D. $\Theta$-stratifications, $\Theta$-reductive stacks and applications, Algebraic geometry: Salt Lake City 2015, Proc. Sympos. Pure Math., 97.1, Amer. Math. Soc., Providence, RI, 2018, 349-379. MR3821155

[16] Jeffrey L, Kirwan F. Localization for nonabelian group actions, Topology 34 (1995), 291-327. MR1318878

[17] Jeffrey L, Kirwan F. Intersection theory on moduli spaces of holomorphic bundles of arbitrary rank on a Riemann surface, Annals of Math. 148 (1998), 109-196. MR1652987

[18] Witten E. Supersymmetry and Morse theory, J. Diff. Geom. 17 (1982), 661-692. MR683171

[19] Witten E. Two-dimensional gauge theories revisited, J. Geom. Phys. 9 (1992), 303-368. MR1185834

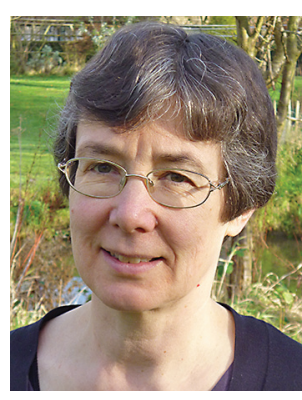

Frances Kirwan

\section{Recollections of Michael Atiyah}

\section{Peter Kronheimer}

Somewhere among my files, I still have the short note that Michael sent me in 1984. I had just completed my undergraduate degree at Oxford, and he was writing to let me know that he would be happy to accept me as a student, beginning in the autumn.

Michael's handwriting sloped upward across the page. The speed and energy of those rising lines was a clear reflection of his personality, which was mirrored in all sorts of other ways: a briefly raised brow as he talked; an abrupt

Peter Kronheimer is the William Caspar Graustein Professor of Mathematics at Harvard University. His email address is kronheim@math . harvard. edu. 
clearing of the throat as he walked down the corridor at the Mathematical Institute; or his forward-leaning stance and searching look, under a slightly asymmetrical frown, as he asked a question, putting a student or colleague on the spot.

It was an exciting time to be working in the closely tied areas of geometry and low-dimensional topology. On the topological side, the three years during which I was a student of Michael's saw the development of Donaldson's polynomial invariants for smooth four-manifolds, Floer's instanton homology for three-manifolds, and the Jones polynomial for knots. On the geometric side, the interactions between Michael Atiyah and Roger Penrose had recently opened up a new area. In particular, the twistor interpretation of "instantons," discovered by Richard Ward, had led to the "ADHM" description of instantons on the four-sphere, named after Atiyah, Drinfeld, Hitchin, and Manin. By the time I was a student, Nigel Hitchin had adapted Ward's work to begin the study of the Bogomolny equations in three-space, solutions of which are Bogomolny monopoles.

The first research paper that Michael asked me to read "in earnest" was Nigel's earliest paper on monopoles, in which he showed how to assign to each solution of the Bogomolny equations a complex-algebraic curve lying on a singular quadric surface. My first-year dissertation was intended to extend part of this story to monopole solutions having simple singularities at prescribed points in threespace. This project introduced me to the rich connections between gauge theory and hyper-Kähler geometry, and it formed a starting point for my doctoral thesis, which established the existence of hyper-Kähler Riemannian metrics on the complex surfaces obtained as resolutions of the Du Val singularities. There was no gauge theory visible on the pages of my thesis, but the ADHM construction lay behind it. My research interests soon swung more towards lowdimensional topology, but the Du Val singularities have never stopped appearing on my blackboard, a constant reference point.

I was fortunate to maintain a close connection with Michael after my degree, first as a postdoc at the Institute for Advanced Study, then as a colleague in Oxford, and then as a regular visitor to Trinity. Meeting with Michael always brought something special. If I parked my small car in the spot marked "Reserved for the Master" and then spent the morning talking mathematics, I knew that I would return home with renewed energy for research and a renewed appreciation for the connectedness of my field.

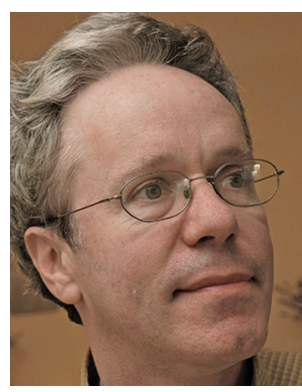

Peter Kronheimer

\section{Recollections of Michael Atiyah}

\section{Ruth Lawrence-Naimark}

Over the three years that I was Atiyah's student, I came for a one-hour meeting regularly once a week during termtime (apart from the semester when he took a sabbatical at IAS and I worked with Nigel Hitchin). To each meeting I would bring a written summary of what I had achieved since the last meeting and would explain it along with obstacles to progress. Despite the fact that he didn't seem to check the details, he was always quick to spot any error. I remember in my first year with him, I worked on a problem that he suggested, a local index theorem. For several weeks, I would come back each week with essentially the same calculation, but with different signs! Finally I got it right, feeling rather embarrassed about the many changes and resolved never to forget the lesson; he was very positive and encouraging, recalling the role of signs in his own work many years earlier.

Atiyah would then usually launch into a private whirlwind tour expounding some relevant topic. Sometimes he would intersperse it with anecdotes from his work with Singer or Bott or his own supervisor, Hodge. One would then feel especially privileged to be hearing about history firsthand. He very rarely directly suggested reading in the literature (although of course one was expected to do background reading); much more often he preferred to give the basics directly and then expect (and recommend) that you worked it out yourself, even if it was already in the literature. Rarely, our discussions moved into areas (like quantum groups) where he didn't feel as comfortable, and then he wouldn't hesitate to suggest talking to an expert in that area.

His Monday afternoon Geometry and Analysis seminar functioned like a departmental colloquium, being the

Ruth Lawrence-Naimark is a professor of mathematics at the Einstein Institute of Mathematics, Hebrew University, Jerusalem. Her email address is ruthe1 @math.huji.ac.i1. 
highlight of the week with often around one hundred attending, some coming from far afield. It was a lecture in this seminar in my second year, by Vaughan Jones, that caught my interest and launched me away from index theory and into knot theory, quantum groups, etc. The following semester Sir Michael was on sabbatical at the Institute in Princeton, which was where Jones-Witten theory was conceived. What is now called Witten-ChernSimons theory had its birth at a dinner at an International Congress in Mathematical Physics in Swansea in July 1988, where Atiyah was pushing the idea of the Jones polynomial arising from a topological quantum field theory, and finally Witten realized that the action needed was precisely Chern-Simons and how it all fit together. (I attended the conference but not that dinner and heard about the event the next day.) Atiyah devoted a whole eight-week term to Jones-Witten theory in the Geometry and Analysis seminar at the start of the following year, with lectures by Segal, Hitchin, and him.

Anyone who has attended a lecture of his can attest to his mastery of subject and audience and his ability to transport you on a beautiful and magical tour of mathematics that would leave you walking out of the lecture feeling that you now understood precisely the interrelations of all these ideas, only to find half an hour later, upon trying to reconstruct the same picture alone, that without his continuing magic touch, the pieces didn't continue to fit so neatly without a lot of extra work!

However, this elusive quality was not there in the personal lectures I had as a student. Sir Michael Atiyah was very practical and down-to-earth, whether about mathematics or logistics, and never left one feeling completely at a loose end. Sure, there was work to be done and unknown factors, but he was a masterful teacher and understood exactly where you were and what you were capable of doing. I remember once visiting Cambridge for a conference, and despite having many demands on his time, he checked that everything was fine with my accommodation and even gave me a private tour of Trinity College (this was a short time before he became Master). He was especially proud showing me the original manuscripts of Sir Isaac Newton in the library.

For him, mathematics had to be unifying and beautiful. I feel very privileged to have been his student and to have had a peek into the view of the glory of mathematics, some fractional part of which I have carried ever since.

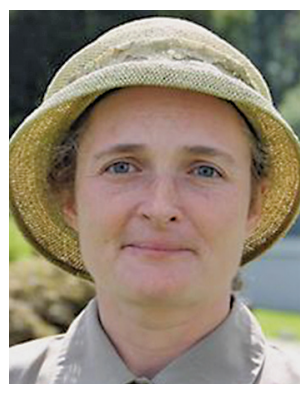

Ruth Lawrence-

Naimark

\section{Memories of Professor Michael Atiyah}

\section{Lisa Jeffrey}

I was the last research student who completed the doctorate under Professor Michael Atiyah's supervision. One of the earliest such students was Graeme Segal, who finished in the sixties and retired more than fifteen years ago from his position as an Oxford mathematics professor. In between, Professor Atiyah supervised more than twenty doctoral students to completion. Most members of the Oxford mathematical community would agree with my belief that if Professor Atiyah had chosen a different profession, many parts of modern mathematics might not have developed.

In the late eighties, Professor Atiyah had encouraged Edward Witten to find an interpretation of the Jones polynomial (an invariant of knots) in terms of quantum field theory. In September 1988, Witten's paper "Quantum field theory and the Jones polynomial" appeared as an IAS preprint. It was published in Communications in Mathematical Physics the following year. The Jones polynomial is associated to a knot in three-space, or in the three-dimensional sphere or a three-dimensional manifold. Witten considered the Chern-Simons functional, which associates a real number to a connection on a three-manifold. He used the Chern-Simons functional as the Lagrangian in a quantum field theory. When a knot in the manifold was specified, one could define the "Wilson loop" (the integral of the connection around the knot) and use this to define a path integral over all connections on the three-manifold. This construction provided the quantum field theory interpretation of the Jones polynomial.

In autumn 1988 the Oxford geometry seminar was devoted to the Oxford seminar on Jones-Witten theory. Ruth

Lisa Jeffrey is a professor of mathematics at the University of Toronto. Her email address is jeffrey@math.toronto.edu. 
Lawrence (at the time a student of Professor Atiyah, completing the final year of her doctorate) wrote notes of the seminar. These were typed (by his extremely efficient secretary, Jane Cox, using an electric typewriter) and made available to participants. The first seminar was given by Professor Atiyah, "An introduction to Jones-Witten theory." Seminar 3 ("Moduli spaces of vector bundles") and Seminar 6 ("The path integral formulation") were also given by Professor Atiyah. Two of the remaining seminars were given by Graeme Segal ("The abelian theory" and "Fusion rules and the Verlinde algebra") and one by Nigel Hitchin ("Reduction to the Abelian case"). Ruth Lawrence gave the final seminar ("Computing the invariants").

In his preface, Professor Atiyah wrote: "No serious attempt has been made to integrate [the notes of the seminars] or produce a coherent polished account. These notes therefore have limited value and are mainly designed for the audience who attended." He is not doing himself justice here. All participants in this seminar felt that the seminar was covering groundbreaking material. The Oxford seminar notes roughly formed the basis for Professor Atiyah's book The Geometry and Physics of Knots (Lezioni Lincee), which was published in 1990. The subject of Jones-Witten theory has had spectacular importance over the more than thirty intervening years, and Professor Atiyah's account has been particularly influential.

I began my doctorate in Oxford in 1988 under Professor Atiyah's supervision. My generation of students was greatly influenced by Professor Atiyah's survey article "New invariants of three- and four-dimensional manifolds," which was published in the proceedings of the 1987 Durham conference The Mathematical Heritage of Hermann Weyl. This article outlined the axioms for topological field theories, which had been formulated by Professors Atiyah and Segal.

It was natural for me to choose a doctoral thesis topic about Jones-Witten theory and its asymptotics (as a perturbative theory with an asymptotic expansion as a sum over contributions coming from flat connections on threemanifolds). However, I was not the only student greatly influenced by the Jones-Witten theory seminar. Joergen Andersen, Andrew Dancer, Oscar Garcia-Prada, Ruth Lawrence, and Michael Thaddeus had the directions of their thesis work altered by this seminar. The research students also spent time on the "junior geometry seminar" (a learning seminar where research students were the speakers) on topics related to the Jones-Witten seminar.

In my notes from an early meeting with Professor Atiyah in early October 1988, the topics covered included flag manifolds, the Peter-Weyl theorem, the Bott-Borel-Weil theorem, and Jones-Witten theory. A subsequent meeting three weeks later covered complex polarization of a sym- plectic manifold, the Grothendieck-Riemann-Roch theorem, the Hirzebruch-Riemann-Roch theorem, derived functors, anomalies, determinant line bundles, Ray-Singer torsion, zeta function regularization, and stationary phase approximation. All of this material was background for the geometry seminar on Jones-Witten theory. My notes for this last meeting filled about ten pages. Professor Atiyah not only listed these topics, he explicated them.

After my first year, Professor Atiyah encouraged me to attend conferences. He told me it might be easier for his students to find an opportunity to talk with him at conferences than back in Oxford, where he had so many responsibilities and preoccupations. I remember the Bonn Arbeitstagung, where Professor Atiyah was always the first speaker. I have a vivid memory of finding a gentian on a German hillside with other mathematicians such as Nigel Hitchin and Graeme Segal.

At one point in my first year I had told Professor Atiyah that I was waiting for an idea to strike. He looked at me rather sternly and told me that ideas do not strike. Perhaps he was referring to Pasteur's statement that chance favors only the well-prepared mind. In any case, it was an immense privilege to work under Professor Atiyah's supervision. I cannot possibly repay the debt of gratitude that I owe him.

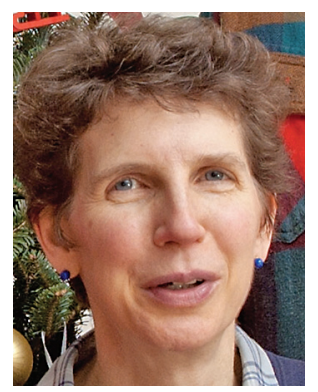

Lisa Jeffrey

\section{Michael Atiyah and Physics: The Later Years}

\section{Bernd Schroers}

The inadvertent and the intentional physicist. Michael Atiyah frequently expressed surprise at the extent to which his work on index theory and his early work in gauge theory turned out to be important in physics. At the end of his commentary in Volume 5 of his collected works he writes:

Bernd Schroers is a professor of mathematics at Heriot-Watt University in Edinburgh. His email address is B.J.Schroers@ahw.ac.uk. 
"...I am really struck by the way most of the work which Singer and I did in the 60s and 70s has become relevant to physics." Reading this work with hindsight one can indeed only marvel at the contrast between the absence of any physical motivation and the ultimate importance in theoretical physics. It seems that, at least during the first half of his mathematical career, Michael's impact on physics was entirely inadvertent. This is also borne out by a remark in his 1998 lecture "The Dirac equation and geometry," where he writes: "When Singer and I were investigating these questions we 'rediscovered' for ourselves the Dirac operator. Had we been better educated in physics, or had there been the kind of dialogue with physicists which is now so common, we would have got there much sooner."

When Michael moved to Edinburgh in 1997, his own physics education had benefited hugely from the dialogue between mathematicians and physicists that he had done so much to initiate and develop. He was now interested in tackling fundamental questions in physics head-on, fully aware of their importance and with the actual intention of revolutionizing the foundations of physics. The inadvertent physicist had become an intentional physicist.

In this brief contribution I will collect some memories and impressions from Michael's second decade in Edinburgh, from about 2009 until his death in 2019. During this time we met regularly, sometimes weekly, for discussions related to the three main projects in physics that Michael pursued: namely, his conjecture regarding the configurations of points in three-dimensional Euclidean space, the role of difference-differential equations in physics, and geometric models of matter.

Our discussions had started because the differencedifferential equations that Michael had introduced in the paper [1] with Greg Moore arise naturally in three-dimensional quantum gravity, which I was working on at the time. However, our focus soon shifted to geometric models of matter, which became the topic of two joint papers $[2,3]$ and a jointly held EPSRC research grant. ${ }^{1}$ I will focus on the geometric models here, but my goal is to identify general themes in Michael's thinking about physics.

The inadequacy of conventional quantum mechanics. Michael expressed his dislike of conventional quantum mechanics on several occasions. He did not believe that any linear theory could be truly fundamental, and he shared Einstein's dislike of the collapse of the wave function induced by observation. He felt that in their efforts to go beyond quantum theory, physicists had tried many avenues but had not critically questioned the paradigm of initial value problems, i.e., the assumption that the future

${ }^{1}$ I believe this is Michael's only EPSRC grant for personal research, i.e., not counting the ones he held as director of the Isaac Newton Institute. fields or wave functions can be computed from values on a particular time slice. The difference-differential equation in [1] abandons that assumption and introduces an element of nonlocality. In his lectures on the subject, Michael highlighted these features as important motivations.

The central role of the Dirac operator. The paper [1] provides an elegant solution to the problem of defining a finite time-shift operator that is also relativistic by exponentiating the Dirac operator. The use of the Dirac operator is no accident. As we already saw, Michael had "rediscovered" this operator in a purely mathematical context, but in his later thinking about physics it always played a central role, often as a possible bridge between the nonlinear world of geometry and the linear world of quantum mechanics. This was also true in the work on geometric models of matter that I discuss next.

The inadequacy of gauge theory and the importance of four-dimensional geometry. Michael's ideas for purely geometric model of particles can be traced back to a 1989 paper with Nick Manton on the Skyrme model in nuclear physics. The paper is based on the observation that one can obtain three-dimensional Skyrme fields by computing the holonomy of instantons in four dimensions, i.e., by integrating along the fourth dimension. Fifteen years later, work by Sakai and Sugimoto suggested that this holonomy can in fact be viewed as the constant term in a Fourier series expansion of an instanton and that the other terms in this expansion can be attributed to further threedimensional meson fields. The message was that threedimensional physics could be described by an $\mathrm{SU}(2)$ gauge theory in four (Euclidean) dimensions.

Michael took this result as an invitation to go one step further. He always felt that gauge theory, the mathematical language in which the standard model of elementary particles is written, required too many arbitrary choices: of a gauge group, of an associated vector bundle, of couplings, and so on. His instinct was therefore to treat the appearance of a fourth dimension as an indication that static, three-dimensional particles could be modelled in terms of the geometry of four-manifolds. Formulating this in detail and studying examples took several years and led to several publications, including [2-4]. The basic challenge, only partly met, was to interpret the (integer) quantum numbers of particle and nuclear physics-like baryon number, lepton number, and electric charge-in terms of topological invariants of four-manifolds. The Dirac operator featured too, its kernel providing the linear space that encodes a particle's spin degrees of freedom.

There is no room here to discuss the geometric models of matter in any detail, but a few general observations about Michael's style of working during the collaboration 
on this topic may be of interest. Even when he was well into his eighties, he worked hard, coming to his office in the School of Mathematics at the University of Edinburgh almost every day that he was not travelling and putting in additional shifts at home. He generated new ideas at a prodigious rate, announcing them with infectious enthusiasm, but abandoning them casually if a new and more promising avenue presented itself. These ideas were essentially mathematical even though the questions we discussed came from physics. Michael needed discussion partners, or at least listeners, to develop his ideas, and he was usually juggling several projects and associated conversations at any point in time. Remarkably, he always took a personal interest in his discussion partners and created additional connections where he could. For example, I was travelling to Africa regularly during our collaboration to teach at one of the African Institutes for Mathematical Sciences (AIMS). Michael immediately offered his help and joined the international advisory board for the AIMS center in Ghana.

Division algebras and Bohmian mechanics. In our discussions, Michael frequently mentioned two other themes that he wanted to incorporate in his description of matter, namely, division algebras and Bohmian mechanics. They never entered his published work on physics in any detail, but I think they are worth recording. Michael felt that the four division algebras-real and complex numbers, quaternions, and octonions - provided essentially the only mathematically natural way to account for the number of fundamental forces (four) or the number of generations (three) in the Standard Model. However, he never settled on a definite proposal of how this matching could work.

Michael liked David Bohm's formulation of quantum mechanics in terms of nonlocal classical variables that follow trajectories determined by the wave function. He felt they clarified some of the foundational issues in quantum mechanics by making the nonlocality explicit, and he hankered after a role for them in his ideas about particles. We discussed a possible use related to his configuration space conjecture but never came up with a convincing proposal.

Passion and beauty. Anybody who interacted with Michael soon noticed that he had a strong personal taste in life and in science and that his instincts were not easily discouraged. Sometimes I felt Michael did not take on board the painstaking work required to match a theoretical model in physics to the experimental data, and occasionally our discussions would turn into arguments. On one such occasion, having listened to Michael's latest ideas, I asked, "But this is just a gut feeling, right?" to which Michael shot back, "Yes, but it is my gut!" Presumably his awareness that his early work, motivated by strictly mathematical considerations, had been so unexpectedly and powerfully relevant to physics boosted his confidence, but I should stress that I never discussed this with Michael.

Michael trusted his own instincts, but he also trusted great minds. He had his heroes, and in physics these were, above all, Maxwell, Einstein, and Dirac. He thought that all their ideas would ultimately prove right in some sense. With Einstein and Dirac he was aware that the work they had done as young men was celebrated, whereas aspects of their later work (e.g., Einstein on unified field theory and Dirac on large numbers) were viewed critically. He felt this was not justified and saw parallels in his own biography.

In an interview for the Newsletter of the European Mathematical Society in November 2018, the last one he gave, Michael offered the following advice to young mathematicians: "What you need is passion, persistence and risking things for searching for the beauty." He certainly lived that advice. Michael's courage to address fundamental questions in science head-on, to follow his own instincts and dreams in seeking answers, and to use beauty as a guide meant that discussions with him transcended the humdrum of scientific activity. Michael sought and encouraged a similar ambition and independence of mind in others, too. In doing so, he took a risk and sometimes attracted criticism, particularly in later years. However, he also created a space for both deep and lateral thinking that is rare and that many treasured.

\section{References}

[1] Atiyah M, Moore G. A shifted view of fundamental physics, Surv. Differ. Geom., 15, Int. Press, Somerville, MA, 2011, pp. 1-15. MR2815723

[2] Atiyah M, Manton NS, Schroers BJ. Geometric models of matter, Proc. Roy. Soc. Lond. A468 (2012), 1252-1279. MR2910348

[3] Atiyah M, Franchetti G, Schroers BJ. Time evolution in a geometric model of a particle, J. High Energy Phys. (2015), 062. MR3321401

[4] Atiyah M, Manton NS. Complex geometry of nuclei and atoms, Internat. J. Modern Phys. A33 (2018), no. 24, 1830022. MR3888339

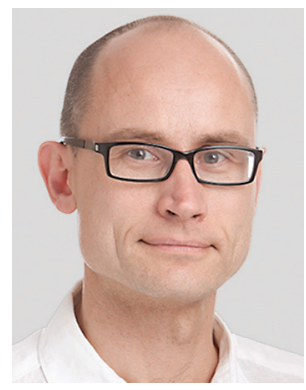

Bernd Schroers 


\section{MEMORIAL TRIBUTE}

\section{Credits}

Figure 1 is from the Atiyah family archives.

Figure 2 and photo of George Lusztig are by Gongqin Li.

Photo of Nigel Hitchin is courtesy of Nigel Hitchin.

Photo of Edward Witten is courtesy of IAS.

Photo of Simon Donaldson is courtesy of Simon Donaldson.

Photo of Frances Kirwan is courtesy of Frances Kirwan.

Photo of Peter Kronheimer is courtesy of Peter Kronheimer.

Photo of Ruth Lawrence-Naimark is courtesy of Archives of the Mathematisches Forschungsinstitut Oberwolfach.

Photo of Lisa Jeffrey is courtesy of Lisa Jeffrey.

Photo of Bernd Schroers is courtesy of Heriot-Watt University

School of Mathematical and Computer Sciences.

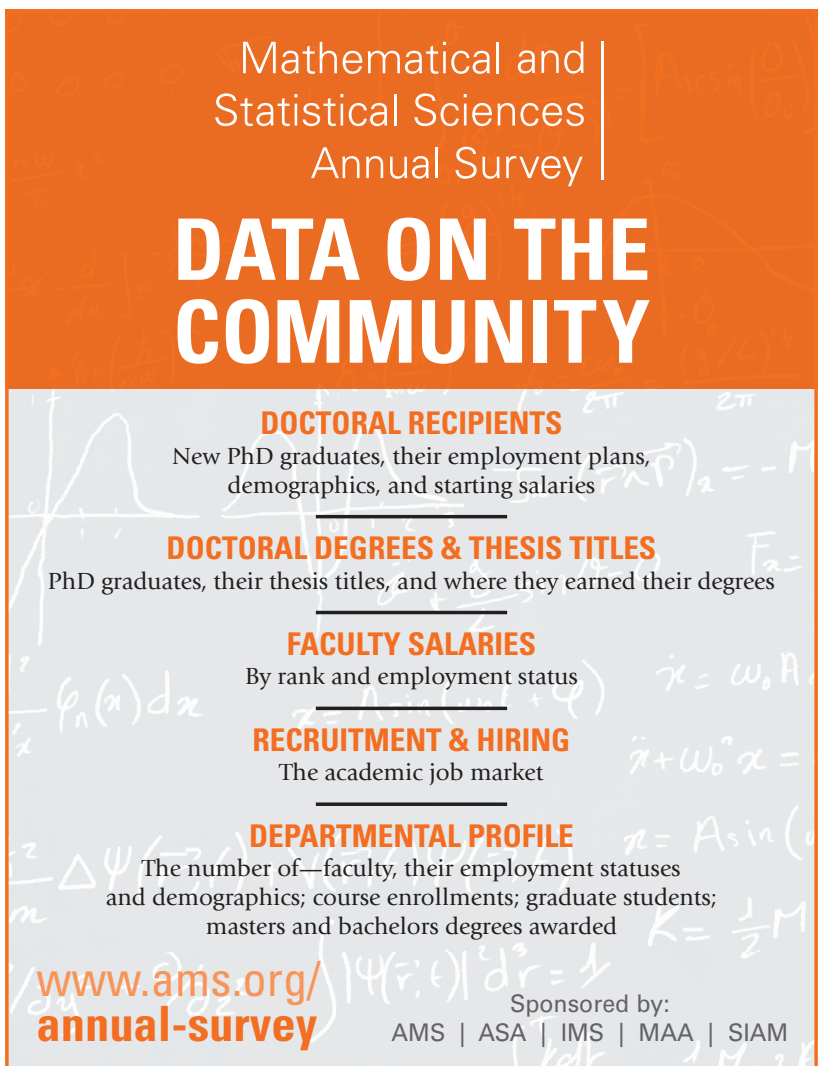

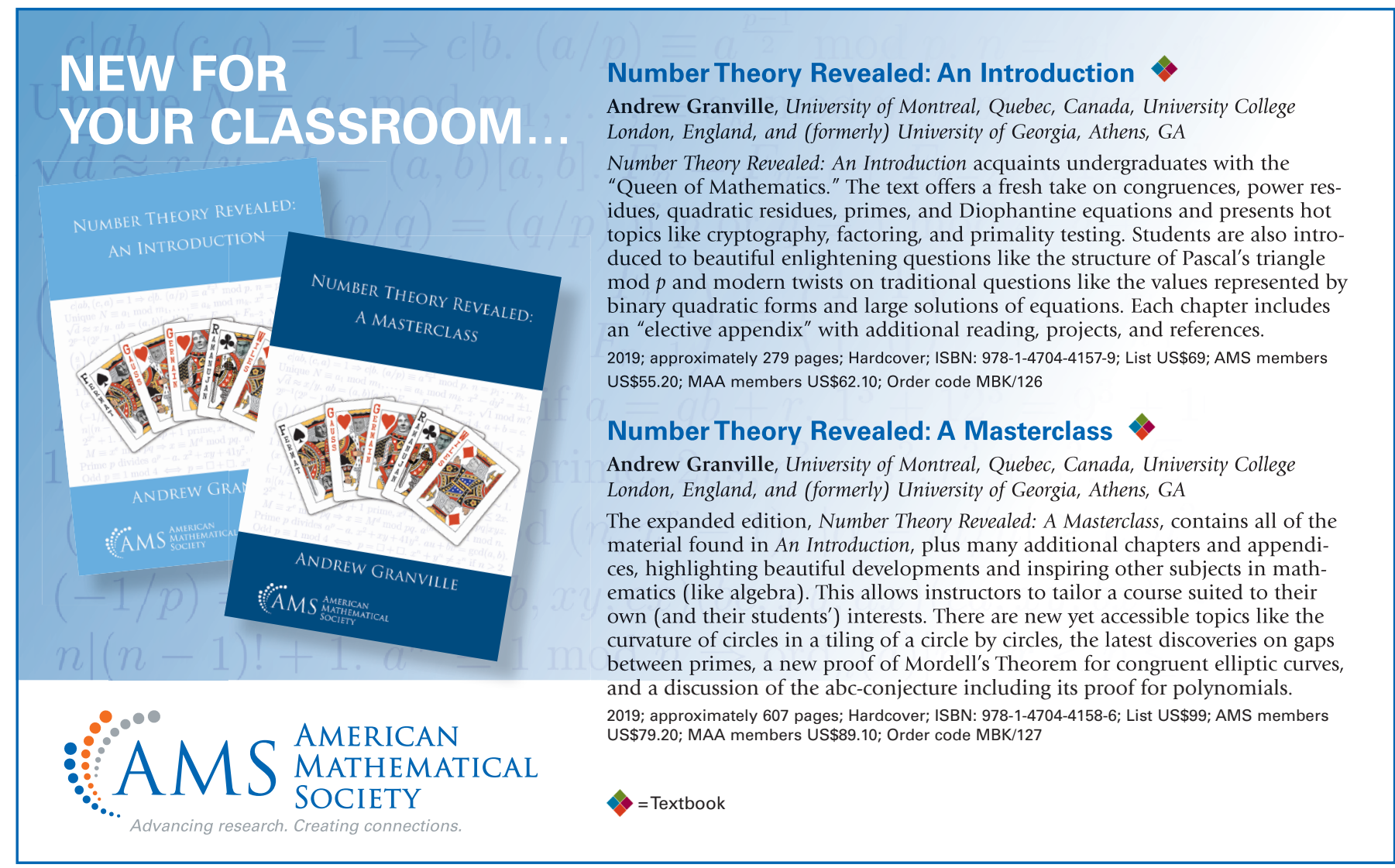

